\title{
Emergency Preparedness 1995 Site Support Plan WBS 6.7.2.3
}

\author{
S. M. Faulk
}

Date Published

September 1994

Prepared for the U.S. Department of Energy

\author{
Westinghouse P.O.Box 1970 \\ (2) Hanford Company Richland, Washington 99352 \\ Hanford Operations and Engineering Contractor for the \\ U.S. Department of Energy under Contract DE-AC06-87RL10930
}

Approved for Public Release

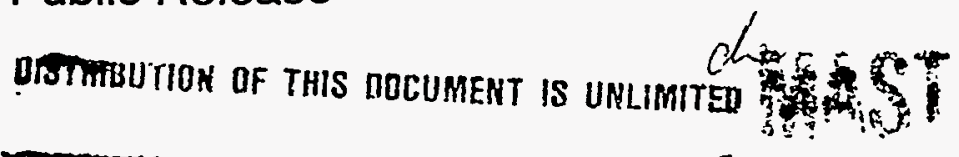




\section{LEGAL DISCLAIMER}

This report was prepared as an account of work sponsored by an agency of the United States Government. Neither the United States Government nor any agency thereof, nor any of their employees, nor any of their contractors, subcontractors or their employees, makes any warranty, express or implied, or assumes any legal liability or responsibility for the accuracy, completeness, or any third party's use or the results of such use of any information, apparatus, product, or process disclosed, or represents that its use would not infringe privately owned rights. Reference herein to any specific commercial product, process, or service by trade name, trademark, manufacturer, or otherwise, does not necessarily constitute or imply its endorsement, recommendation, or favoring by the United States Government or any agency thereof or its contractors or subcontractors. The views and opinions of authors expressed herein do not necessarily state or reflect those of the United States Government or any agency thereol.

This report has been reproduced from the best available copy. Available in paper copy and microfiche.

Available to the U.S. Department of Energy and its contractors from

Office of Scientific and Technical Information

P.O. Box 62

Oak Ridge, TN 37831

(615) 576-8401

Printed in the United States of America 


\section{DISCLAIMER}

Portions of this document may be illegible in electronic image products. Images are produced from the best available original document. 


\section{RELEASE AUTHORIZATION}

\begin{tabular}{ll} 
Document Number: & WHC-SP-1107 \\
\hline Document Title: & $\begin{array}{l}1995 \text { Site Support Program PTan - Emergency } \\
\text { Preparedness }\end{array}$
\end{tabular}

Release Date: $\quad 8 / 25 / 94$

***************

This document was reviewed following the procedures described in WHC-CM-3-4 and is:

APPROVED FOR PUBLIC RELEASE

*************

WHC Information Release Administration Specialist:

Chisterw Hellerigham
c. Wiltingham

$8 / 25 / 94$

(Signature)

$\frac{8 / 25 / 94}{\text { (Date) }}$




\title{
Site Support Program Plan Approval Sheet
}

\author{
EMERGENCY PREPAREDNESS - WBS \#6.7.2.3
}

Assistant Manager-Contracting Officer's Representative

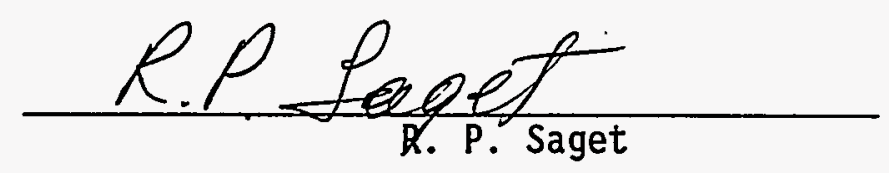

$\frac{9 / 23 / 94}{\text { Datef }}$

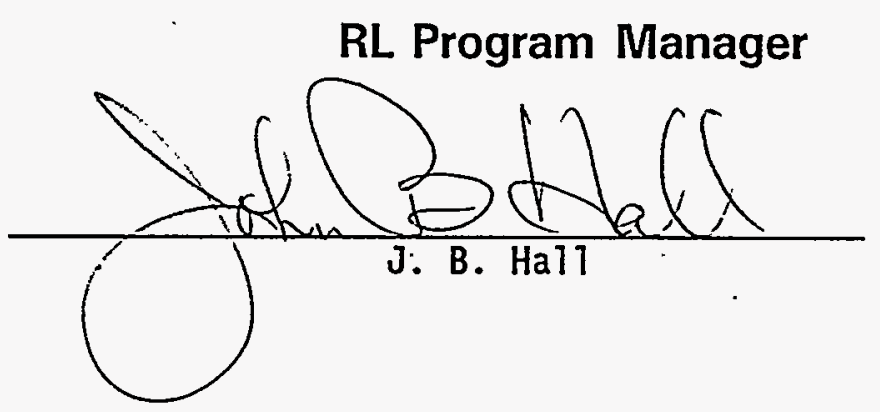

$$
\frac{9.19 .944}{\text { (Date) }}
$$

WHC Director

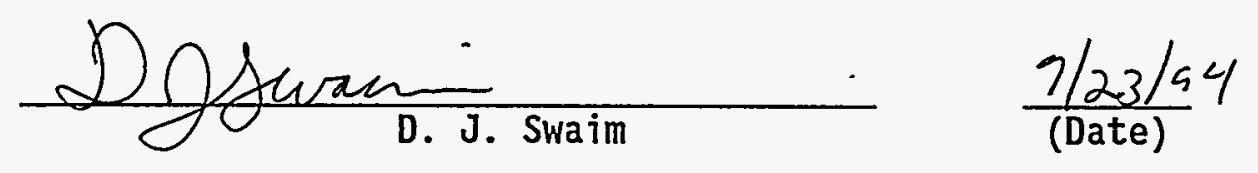

WHC Program Manager

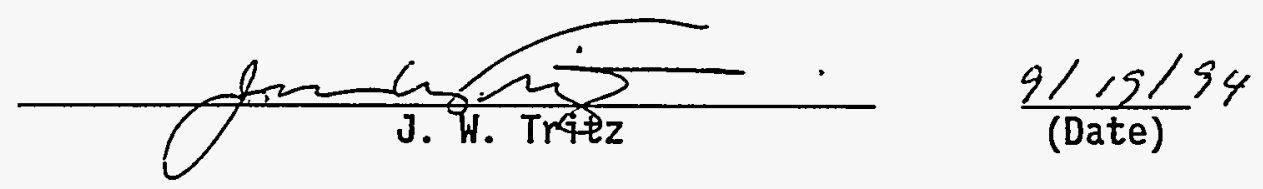




\section{EMERGENCY PREPAREDNESS \\ 1995 \\ SITE SUPPORT PROGRAM PLAN \\ WBS \# 6.7.2.3 \\ TABLE OF CONTENTS}

\subsection{OVERVIEW}

Number Title

Page

1.A Vision/Mission

1.B.1 Internal Assessment Summary

1

1.B.2

External Assessment Summary

2

1.C

Goals and Objectives

1.D

Strategies

1.E

Assumptions

1.F

Issues and Constraints

11

1.G

Performance Measures

\subsection{BASELINES}

Number $\quad$ TitTe

2.A.1 WBS \& Responsibility Assignment Matrix

2.A.2 Description of Activities

2.B.1 Milestone List

16

2.C. 1.1

Cost Baseline by Funding Source

18

2.C. 3

FTE Forecasts

\subsection{EXECUTION YEAR PACKAGE}

Title

Dictionary Sheets

Milestone Description Sheets 

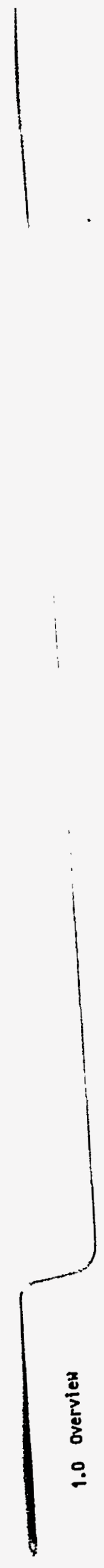

- . A 


\begin{tabular}{|c|c|c|}
\hline 1.A Vision/Mission & $\begin{array}{c}\text { Westinghouse Hanford Company } \\
\text { Emergency Preparedness } \\
6.7 .2 .3\end{array}$ & $\begin{array}{c}\text { FY 1995 } \\
\text { Site Support Program Plan } \\
\text { Date Prepared: } 8 / 31 / 94\end{array}$ \\
\hline
\end{tabular}

The Emergency Preparedness Program provides an emergency management system including occurrence notification; development, coordination, and direction of planning, preparedness, and readiness assurance for response to emergency events on the Hanford Site; and emergency management support to Department of Energy, Richland Operations Office (RL). 


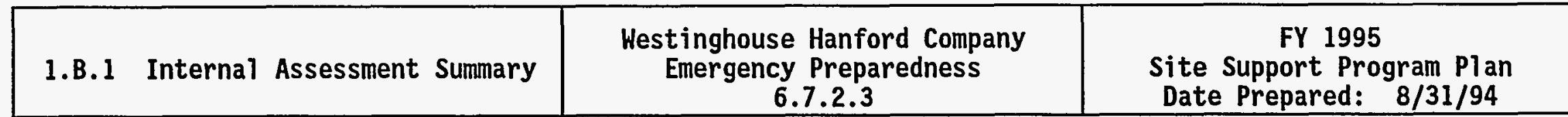

\section{PRIMARY CUSTOMERS}

DOE RL

WHC Facilities

PNL

BHI

HEHF

\section{PRODUCTS/SERVICES PROVIDED}

\section{Hazard Assessments}

Emergency Plans and Procedures

Emergency Response Organization

Emergency Facilities and Equipment

Emergency Preparedness Training

Emergency Preparedness Drills and Exercises

Emergency Readiness Appraisals

Emergency Readiness Assurance Plan

Offsite Interface Assistance

Occurrence Reporting 


\section{B.1 Internal Assessment Summary}

Westinghouse Hanford Company Emergency Preparedness 6.7 .2 .3
FY 1995

Site Support Program Plan Date Prepared: 8/31/94

\section{MAJOR ACTIVITIES PERFORMED TO DELIVER PRODUCTS. AND SERVICES}

The Hanford Site Emergency Preparedness program implements the DOE emergency management orders, occurrence reporting orders, as well as applicable state and federal regulations to ensure the health and safety of Site employees and the general public. These efforts include hazards assessments that form the basis of the emergency management program, planning to ensure integrated onsite emergency response, planning with state and local agencies to ensure that the public can be protected, developing and maintaining emergency plans and procedures, staffing and maintaining emergency response facilities, notifications and occurrence reporting, maintaining the readiness of emergency response organization through training and drill, and validating the Site Emergency Management program through exercises and evaluation. The primary activities associated with this include:

\section{Hazards Assessment}

Hazards Assessments activities provide the basis for the emergency management program and provide consequence assessment capability to protect Site personnel and make offsite protective action recommendations during operational emergencies. Specific activities include:

Develop and revise hazards assessments.

Staff and maintain Unified Dose Assessment Center (UDAC).

Maintain readiness of UDAC emergency response organization.

Provide technical interface assistance to $\mathrm{RL}$ with state, local, federal and tribal emergency response agencies.

Develop and revise procedures to ensure UDAC emergency response organization can provide consequence assessment information to onsite and offsite emergency response organizations. 


\section{B.1 Internal Assessment Summary \\ Westinghouse Hanford Company \\ Emergency Preparedness \\ 6.7 .2 .3 \\ FY 1995 \\ Site Support Program P1an \\ Date Prepared: 8/31/94}

\section{Integration, Assessment and RL Support}

Integration, Assessment and RL Support ensures that onsite planning and offsite planning with state and local agencies take place so that an adequate emergency management program is in place. It maintains plans and procedures to document emergency response activities. It ensures that an adequate program is in place through appraisals and maintains the readiness of RL emergency response facilities and organizations. Specific activities include:

Develop and maintain RL and WHC emergency plans and procedures.

Provide interface assistance to RL with state, local, federal and tribal emergency response agencies.

Staff and maintain the RL Emergency Operations Center (RL EOC) and alternate center.

Maintain readiness of the RL EOC emergency response organization.

Coordinate and perform facility emergency readiness appraisals.

Coordinate development of the Emergency Readiness Assurance Plan.

Coordinate WHC Emergency Preparedness Site Support Program Planning.

Develop and revise procedures to ensure RL EOC emergency response organization can manage emergency, protect personnel and provide protective action recommendation to offsite agencies.

The Hanford Site Exercise Program is also a part of the Integration, Assessment and RL Support organization. This program exercises the Site emergency response organization and state and local emergency response organization to ensure that they can adequately respond to an operational emergency to protect the health and safety of Site personnel and offsite populations. Specific activities include:

Develop and coordinate the Hanford Site field exercises including working with state, local, federal and tribal emergency response agencies to exercise with them. 


\section{B.1 Internal Assessment Summary}

Westinghouse Hanford Company

Emergency Preparedness

6.7 .2 .3

FY 1995

,

Develop and coordinate Site limited exercises.

Develop, coordinate and conduct monthly RL-EACT drill sessions.

\section{Training and Area Support}

Training and Area Support maintains the readiness of the Site emergency response facilities and organizations to mitigate operational emergencies and provide onscene emergency response and area coordination. These efforts are also critical in ensuring that initial notifications and protective action recommendations are provided to offsite agencies. Specific activities include:

Develop material and coordinate training to maintain readiness of emergency response personnel.

Staff and maintain area emergency control centers.

Maintain readiness of the facility and area emergency response organizations

Provide assistance and oversight to ensure facility emergency response organization readiness.

Develop and revise procedures to ensure facility and area emergency response organizations can manage emergency and protect personnel.

\section{Occurrence Notification Center}

The Occurrence Notification Center activities implement the requirements of DOE Order 5000.3B and provides regulatory and emergency notifications to local, state and federal agencies. Activities include:

Occurrence Reporting in accordance with DOE Order 5000.3B.

Occurrence Report services to all site contractors and RL.

Regulatory reporting and notifications. 
Notifications to offsite agencies during operational emergencies for event classification and protective action recommendations in accordance with DOE Order 5500.2B.

Occurrence Reporting Training for the Site.

\section{EVALUATION OF MAJOR ACTIVITIES (COST, VALUE-ADDED, ETC.)}

The Hanford Site Emergency Preparedness Program is a cost effective program that maintains the readiness to deal with an operational emergency in order to be able to protect the health and safety of the workforce and the general public as required by the DOE 5500 series orders. The organization is constantly striving to improve performance while looking for ways to reduce cost and streamlining operations. Examples of cost savings in the past several years include the take over of the workscope in assisting RL, which was a significant cost savings to the Site, assessment of emergency center needs and restructuring when possible, reducing the scope of the EOC Integration Project, reduction of emergency preparedness requirements for non hazardous facilities, and consolidation of staff responsibilities. There have been many significant improvements in the program, including:

Full implementation of the revised DOE 5500 Series orders without significant additional costs.

Development of a the Emergency Management System, a significant consequence assessment tool.

Streamlining the Emergency Management Organization by combining several Site organizations.

Developing and implementing the Hanford Site Emergency Response Plan

Developing and conducting a full scope ingestion exercise including exercising with the Federal Radiological Monitoring and Assessment Center which was a first in the DOE complex.

Working with Management Standards and Document Control to revise the way in which procedures are issued so that emergency response organizations members have only the procedures which specifically pertain to them. 


\begin{tabular}{|c|c|c|c|}
\hline $1 . B .1$ & Internal Assessment Summary & $\begin{array}{c}\text { Westinghouse Hanford Company } \\
\text { Emergency Preparedness } \\
6.7 .2 .3\end{array}$ & $\begin{array}{l}\text { FY } 1995 \\
\text { Site Support Program Plan } \\
\text { Date Prepared: } 8 / 31 / 94\end{array}$ \\
\hline
\end{tabular}

Emergency Preparedness will continue to look for ways to improve the program in 1995 and beyond as well a always being vigilant for opportunities to streamline operations and save money.

\section{FACTORS WHICH INFLUENCE RESOURCE CONSUMPTION (CHANGES TO COST AND VOLUME}

Emergency Preparedness is currently maintaining a program that meets the requirements of the D0E 5500 Series orders and the expectations of its customers including RL, DOE HQ, and the offsite agencies. The factors that will change resource consumption is a change to the orders or a change in customer expectations which is not expected to happen in 1995.

\section{FACTORS THAT INFLUENCE CHANGES IN TYPES OR NATURE OF PRODUCTS and SERVICES}

See above. 


\section{B.2 External Assessment Summary}

Westinghouse Hanford Company

Emergency Preparedness

6.7.2.3

\section{SUMMARY OF CUSTOMER REQUIREMENTS (NEEDS)}

\section{$\underline{\mathrm{RL}}$}

Completion of the EOC Integration Project

Maintenance of Emergency Facilities and equipment

Maintaining a trained emergency response organization

Assistance with offsite interface

$P 7$ an and procedure revision

Periodic exercises to validate program

Coordination of Emergency Readiness Assurance Plan

\section{Facilities}

\section{Emergency Preparedness Training} Drills

Appraisals to assess program Emergency Equipment

Periodic exercises to validate program

Hazards Assessment revisions

Emergency Preparedness Procedures

occurrence Reporting and Processing Assistance

\section{OTHER EXTERNAL CHALLENGES}

\section{None}




\begin{tabular}{|l|c|c|}
\hline 1.C Goals and Objectives & $\begin{array}{c}\text { Westinghouse Hanford Company } \\
\text { Emergency Preparedness } \\
6.7 .2 .3\end{array}$ & $\begin{array}{c}\text { FY 1995 } \\
\text { Site Support Program Plan } \\
\text { Date Prepared: 8/31/94 }\end{array}$ \\
\hline
\end{tabular}

\section{Program Goals and Objectives}

The 1995 objectives of the Westinghouse Hanford Company (WHC) Emergency Preparedness Program are to maintain compliance with the DOE Orders for emergency preparedness and occurrence reporting and the Emergency Management Guidelines.

The program will continue to provide quality products and services and continue to enhance the Site Emergency Preparedness Program.

\section{Program Priorities}

In addition to the activities described in Section 1B1, there are some specific priorities in improving the emergency management system at the Hanford Site. The priorities are 1isted below and will be met by completion of the specific RL and contractor milestones described in the Milestone Description Sheets.

1. Complete Emergency Operations Center Integration Project.

2. Complete the activities associated with the Hanford Site Emergency Exercise Program.

3. Improve the Occurrence Reporting System.

4. Enhance the offsite interface program.

5. Enhance the Site hazards assesesment and consequence assessement capabilities.

6. Develop a Transportation Emergency Preparedness Program 


\begin{tabular}{|c|c|c|c|}
\hline I.D & Strategies & $\begin{array}{c}\text { Westinghouse Hanford Company } \\
\text { Emergency Preparedness } \\
6.7 .2 .3\end{array}$ & $\begin{array}{l}\text { FY } 1995 \\
\text { Site Support Program P1an } \\
\text { Date Prepared: } 8 / 31 / 94\end{array}$ \\
\hline
\end{tabular}

\section{CUSTOMER SUPPORT STRATEGIES}

The strategy to support the customers is to understand their needs and be able to respond to them. In order to do this, weekly meetings, monthly meetings, and quarterly meetings are held to ensure that we understand what the customers are asking for and that they are cognizant of and approve our activities.

\section{PRODUCTION STRATEGIES}

Emergency Preparedness is continuously looking to make improvements to the way it does business. It establishes goals at the start of each year and focusses on self assessment to ensure that progress is being made towards those goals and to review any changes that may require changes or additional goals throughout the year.

\section{ORGANIZATION and MANAGEMENT STRATEGIES}

The Emergency Preparedness Organization has undergone significant changes the past two years in order to respond to additional requirements, customer needs, and company organizational strategies. The current organizational structure is working and close to optimum. No change in organizational structure is anticipated in 1995. 


\begin{tabular}{|c|c|c|}
\hline 1.E Assumptions & $\begin{array}{c}\text { Hestinghouse Hanford Company } \\
\text { Emergency Preparedness } \\
6.7 .2 .3\end{array}$ & $\begin{array}{c}\text { FY } 1995 \\
\text { Site Support Program Plan } \\
\text { Date Prepared: } 8 / 31 / 94\end{array}$ \\
\hline
\end{tabular}

Facility status will not cause a change to Emergency Preparedness support during FY 1995.

No significant changes to DOE Orders and federal regulations are anticipated that will impact the Hanford Site beyond this plan. 


\begin{tabular}{|c|c|c|}
\hline 1.F Issues and Constraints & $\begin{array}{c}\text { Westinghouse Hanford Company } \\
\text { Emergency Preparedness } \\
6.7 .2 .3\end{array}$ & $\begin{array}{c}\text { FY } 1995 \\
\text { Site Support Program Plan } \\
\text { Date Prepared: } 8 / 31 / 94\end{array}$ \\
\hline
\end{tabular}

None 


\section{G Performance Measures}
Westinghouse Hanford Company
Emergency Preparedness 6.7 .2 .3

FY 1995

Site Support Program P1an Date Prepared: 8/31/94

\section{Emergency Preparedness Performance Measures}

Assessment of RL and WHC Milestones on Milestone Schedule

Performance measures on level of effort activities include:

Building Emergency Director Training

Dril1 Completed

Emergency Control Center Training

Building Emergency Plan Revisions

Surveillances Conducted

occurrence Reporting

All significant improvement items are tracked using Quality Improvement Action Plans which are statused periodically by management and bi monthly at Emergency Preparedness Program Reviews. 
i.

:

.

)

1

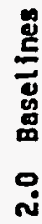

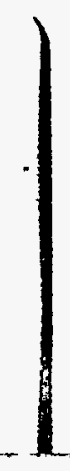




\begin{tabular}{|c|c|c|c|c|c|}
\hline \multicolumn{2}{|c|}{$\begin{array}{c}\text { 2.A.1 } \\
\text { WBS \& Responsibility } \\
\text { Assignment } \\
\text { Matrix }\end{array}$} & \multicolumn{2}{|r|}{$\begin{array}{c}\text { Westinghouse Hanford Company } \\
\text { Emergency Preparedness } \\
6.7 .2 .3\end{array}$} & \multicolumn{2}{|c|}{$\begin{array}{c}\text { FY } 1995 \\
\text { Site Support Program Plan }\end{array}$} \\
\hline $\begin{array}{l}\text { Activity } \\
\text { No. }\end{array}$ & $\begin{array}{c}\text { Cost } \\
\text { Account }\end{array}$ & $\begin{array}{c}\text { Hork } \\
\text { Package No. }\end{array}$ & Title & $\begin{array}{l}\text { Responsible } \\
\text { Manager }\end{array}$ & $\begin{array}{l}\text { Responsibb } \\
\text { Organizatiot }\end{array}$ \\
\hline 6.7 .2 .3 & & & Emergency Preparedness & $J H$ Tritz & 3A000 \\
\hline 6.7 .2 .3 .1 & & & Occurrence Notification Center & DJ Connell & उA100 \\
\hline 6.7 .2 .3 .2 & & & Emergency Management & JW Tritz & \\
\hline & & & & & \\
\hline & & & & & \\
\hline & & & & & \\
\hline & & & & & \\
\hline & & & & & \\
\hline
\end{tabular}




\begin{tabular}{|c|c|c|}
\hline $\begin{array}{l}\text { 2.A.2 Description } \\
\text { of Activities }\end{array}$ & \multicolumn{2}{|c|}{$\begin{array}{c}\text { Westinghouse Hanford Company } \\
\text { Emergency Preparedness } \\
6.7 .2 .3\end{array}$} \\
\hline Activity No. & Activity Title & Description \\
\hline 6.7 .2 .3 .1 & Occurrence Notification Center & $\begin{array}{l}\text { Activities include: Occurrence Reporting in } \\
\text { accordance with DOE Order 5000.3B. Occurrence Report } \\
\text { services to all Site contractors and RL. Regulatory } \\
\text { reporting and notifications. Notifications to } \\
\text { offsite agencies during operational emergencies for } \\
\text { event classification and protective action } \\
\text { recommendations in accordance with DOE Order } \\
5500.2 \mathrm{~B} \text {. Occurrence Reporting Training for the } \\
\text { Site. }\end{array}$ \\
\hline 6.7 .2 .3 .2 & Emergency Management & $\begin{array}{l}\text { Activities include: Develop and revise hazards } \\
\text { assessments. Develop and coordinate the Hanford } \\
\text { Site field exercises including working. with state, } \\
\text { local, federal and tribal emergency response } \\
\text { agencies to exercise with them.Develop and } \\
\text { coordinate Site limited exercises. Develop, } \\
\text { coordinate and conduct monthly RL-EACT dril1 } \\
\text { sessions. Develop and maintain RL and WHC emergency } \\
\text { plans and procedures. Provide interface assistance } \\
\text { to RL with state, local, federal and tribal } \\
\text { emergency response agencies. Staff and maintain the } \\
\text { Site emergency response facilities. } \\
\text { Maintain readiness of the Site emergency response } \\
\text { organizations. Coordinate and perform facility } \\
\text { emergency readiness appraisals. Coordinate } \\
\text { development of the Emergency Readiness Assurance } \\
\text { Plan. Develop material and coordinate training to } \\
\text { maintain readiness of emergency response personnel. } \\
\text { Provide assistance and oversight to ensure facility } \\
\text { emergency response organizaition readiness. }\end{array}$ \\
\hline
\end{tabular}




\begin{tabular}{|c|c|c|c|c|}
\hline \multicolumn{3}{|c|}{ 2.B.1 Milestone List } & $\begin{array}{c}\text { Westinghouse Hanford Company } \\
\text { Emergency Preparedness } \\
6.7 .2 .3\end{array}$ & $\begin{array}{c}\text { FY } 1995 \\
\text { Site Support Program Plan } \\
\text { Date Prepared: } 8 / 31 / 94 \\
\end{array}$ \\
\hline \multicolumn{2}{|c|}{ Milestone } & \multirow{2}{*}{$\begin{array}{c}\text { WBS } \\
\text { Number }\end{array}$} & \multirow{2}{*}{ Milestone Description } & \multirow{2}{*}{$\begin{array}{c}\text { Scheduled } \\
\text { Completion } \\
\text { Date } \\
\end{array}$} \\
\hline Type & Number & & & \\
\hline $\mathrm{RL}$ & HEP-95-001 & 6.7.2.3 & Implement Emergency Operations Center Project & $1 / 31 / 95$ \\
\hline WHC & HEP-95-002 & 6.7.2.3 & Complete Construction of Emergency Operations Center & $1 / 01 / 95$ \\
\hline WHC & HEP-95-003 & 6.7 .2 .3 & Complete New Emergency Operations Center Procedures & $1 / 01 / 95$ \\
\hline WHC & HEP-95-004 & 6.7 .2 .3 & Complete Emergency Operations Center Personnel Training & $1 / 31 / 95$ \\
\hline $\mathrm{RL}$ & HEP-95-005 & 6.7 .2 .3 & Complete Hanford Site Emergency Exercise Program & $7 / 31 / 95$ \\
\hline WHC & HEP-95-006 & 6.7 .2 .3 & Revise the Exercise Evaluation Procedure & $1 / 31 / 95$ \\
\hline WHC & HEP-95-007 & 6.7 .2 .3 & Track Exercise Participation & $3 / 31 / 95$ \\
\hline WHC & HEP- $95-008$ & 6.7 .2 .3 & Complete major Site field Exercise & $7 / 31 / 95$ \\
\hline $\mathrm{RL}$ & HEP-95-009 & 6.7.2.3 & Improve Occurrence Reporting System & $9 / 30 / 95$ \\
\hline WHC & HEP-95-010 & 6.7.2.3 & Improve ORPS Communications Access & $9 / 30 / 95$ \\
\hline WHC & HEP-95-011 & 6.7.2.3 & Implement Procedure Change Based on 5000.3B Change & Variable \\
\hline WHC & HEP-95-012 & 6.7.2.3 & Implement ORPS Software and Database Changes & Variable \\
\hline WHC & MEP-95-013 & 6.7.2.3 & Revise QTRC Occurrence Reporting Training Program & Variable \\
\hline WHC & HEP-95-014 & 6.7 .2 .3 & Develop Assessment/Oversight Plan for Occurrence Reporting & $7 / 31 / 95$ \\
\hline $\mathrm{RL}$ & HEP-95-015 & 6.7 .2 .3 & Enhance Offsite Interface Program & $9 / 30 / 95$ \\
\hline WHC & HEP-95-016 & 6.7.2.3 & Develop Electronic Notification Form & $1 / 31 / 95$ \\
\hline WHC & HEP-95-017 & 6.7.2.3 & Fund Emergency Public Information Calender & $9 / 30 / 95$ \\
\hline
\end{tabular}









\begin{tabular}{|c|c|c|}
\hline 2.C.1.1 & Westinghouse Hanford Company & Fy 1995 \\
Cost Baseline by Funding Source & $\begin{array}{c}\text { Site Support Program P1an } \\
\text { D.7.2.3 }\end{array}$ & $\begin{array}{c}\text { Site Prepared: } 8 / 31 / 94 \\
\text { Datess }\end{array}$ \\
\hline
\end{tabular}

\begin{tabular}{|l|l|c|c|c|c|c|}
\hline \multicolumn{1}{|c|}{ WBS \# } & \multicolumn{1}{|c|}{ Title 1994 Cost Baseline (Dollars in 000s) } & SLP & G A & Direct \$ & Total \$S & Dept. OH \\
\hline 6.7 .2 .3 .1 & $\begin{array}{l}\text { Occurrence Notification } \\
\text { Center }\end{array}$ & 720.0 & & & \\
\hline 6.7 .2 .3 .2 & Emergency Management & 4690.0 & & & \\
\hline & & & & & & \\
\hline & & & & & & \\
\hline & & & & & & \\
\hline
\end{tabular}

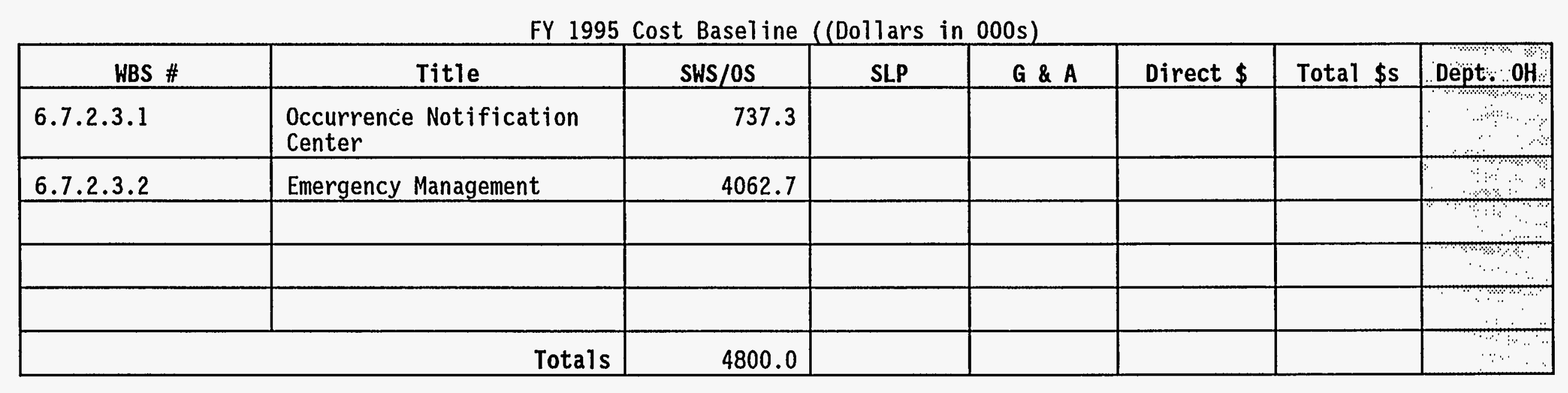




\begin{tabular}{|c|c|c|}
\hline 2.C.1.1 & Westinghouse Hanford Company & FY 1995 \\
Cost Baseline by Funding Source & Emergency Preparedness \\
6.7 .2 .3 & $\begin{array}{c}\text { Site Support Program Plan } \\
\text { Date Prepared: } 8 / 31 / 94\end{array}$ \\
\hline
\end{tabular}

\begin{tabular}{|l|l|c|c|c|c|c|}
\hline \multicolumn{1}{|c|}{ WBS \# } & \multicolumn{1}{|c|}{ Title 1996 Cost Baseline (Dollars in 000s) } & SLP & G \& A & Direct \$ & Total \$s & Dept. OH \\
\hline 6.7 .2 .3 .1 & $\begin{array}{l}\text { Occurrence Notification } \\
\text { Center }\end{array}$ & 758.7 & & & \\
\hline 6.7 .2 .3 .2 & Emergency Management & 4180.5 & & & \\
\hline & & & & & & \\
\hline & & & & & & \\
\hline & & & & & & \\
\hline & & & & & & \\
\hline
\end{tabular}

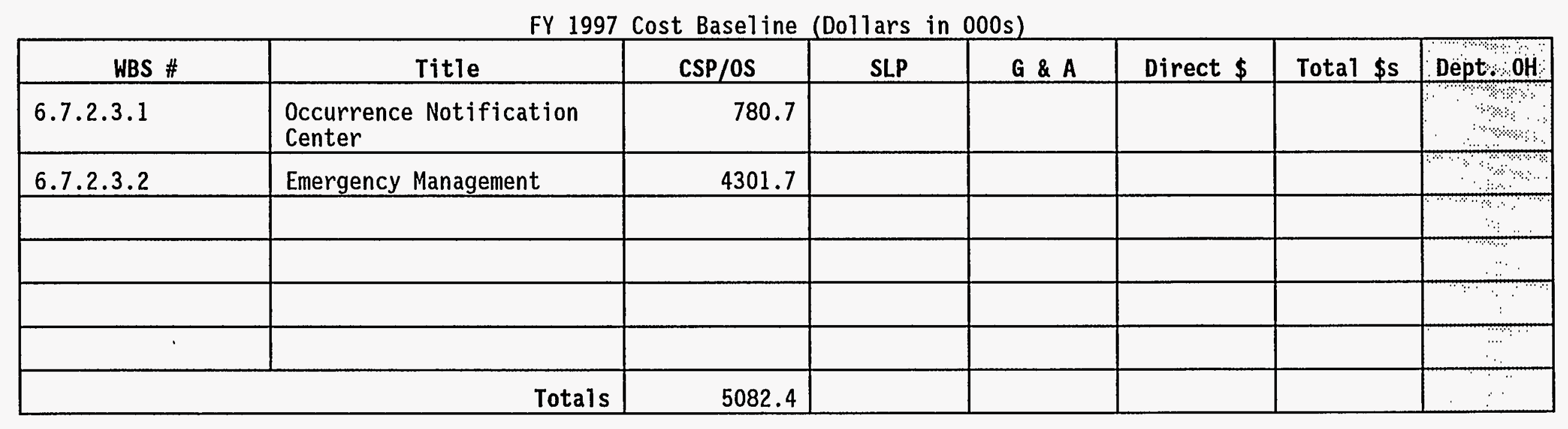




\begin{tabular}{|c|c|c|c|c|c|c|c|c|}
\hline 2.C.3 FTE Forecasts & \multicolumn{4}{|c|}{$\begin{array}{c}\text { Hestinghouse Hanford Company } \\
\text { Emergency Preparedness } \\
6.7 .2 .3\end{array}$} & & \multicolumn{3}{|c|}{$\begin{array}{c}\text { FY } 1995 \\
\text { Site Support Program Plan } \\
\text { Date Prepared: } 8 / 31 / 94\end{array}$} \\
\hline \multicolumn{9}{|c|}{ JOB FAMILY - FuTI Time Equivalent Staff by Job Descriptjon } \\
\hline Job category & 1994 & 1995 & 1996 & 1997 & 1998 & 1999 & 2000 & 2001 \\
\hline \multicolumn{9}{|c|}{ MANAGERS } \\
\hline First line & 5.0 & 4.0 & 4.0 & & $\because$ & $\because$ & 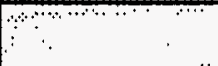 & \\
\hline General/executive & 1.0 & 1.0 & 1.0 & & an & $\ldots$ & $\because$ & $\therefore$ \\
\hline Project/Program & & & & & a & : & अ & \\
\hline Other & & & & किखि & अनि & मे। & \%ै\% & $\because: \because \cdots$ \\
\hline Subtotal Managers & 6.0 & 5.0 & $\overline{5.0}$ & 5.0 & 5.0 & 5.0 & 5.0 & 5.0 \\
\hline \multicolumn{9}{|c|}{ ENGINEERS $\quad$ U } \\
\hline Chemical & & & & $\because \because \frac{\ddots}{3}$ & औ & $\therefore$ - $\quad \therefore<\cdots$ & н. & $\because$ \\
\hline \multicolumn{9}{|c|}{ A } \\
\hline \multicolumn{9}{|l|}{ Computer Software } \\
\hline Electrical & & & & में : & ঋै। - । & औै। & स, ब, & $\because \quad \cdots \quad$ : \\
\hline Environmental & & & & अै। मे। & अ & के & a $\quad \cdots$ & a \\
\hline Industrial & & & & r & \% $\quad$ on & : & o & 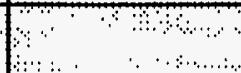 \\
\hline Mechanical & & & & मे & . & $\because \quad \therefore \quad \therefore$ & \begin{tabular}{lll}
0 & \\
\hdashline & $\vdots$
\end{tabular} & \\
\hline Nuclear & & & & a & 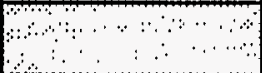 & 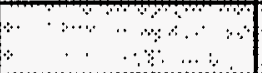 & $\cdots$ & \\
\hline Plant & & & & : : & u & & & $\therefore$ \\
\hline Qual ity Control & & & & 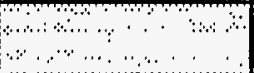 & & 18 & & $\therefore$ \\
\hline Safety & & & & & $\sqrt{x}$ & & & \\
\hline other & 1.0 & 1.0 & 1.0 & . & & & & \\
\hline Subtotal Engineers & 1.0 & 1.0 & 1.0 & 1.0 & 1.0 & 1.0 & 1.0 & 1.0 \\
\hline
\end{tabular}




\begin{tabular}{|c|c|c|c|c|c|c|c|c|}
\hline $0^{\circ} \varepsilon 2$ & $0 \cdot \varepsilon 2$ & $0^{\circ} \varepsilon ट$ & $0^{\circ} \Sigma \Sigma$ & $0 \cdot \varepsilon 2$ & $0 \cdot \varepsilon \Sigma$ & $0^{\circ} \varepsilon \Sigma$ & $0 \cdot \varepsilon 2$ & sjeuo!ssajosd Jayzo/u!upy jezozqns \\
\hline & . & . & & & $0 \cdot \varepsilon 2$ & $0 . \varepsilon 2$ & $0 \cdot \varepsilon 2$ & Јอบ70 \\
\hline & $\therefore$ & & $\therefore \quad \therefore \quad \cdots$ & $\left|\begin{array}{lll}0 & 0\end{array}\right|$ & & & & ssau!ed1 \\
\hline & $\therefore \therefore$ & $\cdots$ & $\therefore \quad \therefore \quad \cdots$ & $\begin{array}{lll}0 & \vdots\end{array}$ & & & & A 4!.Jnכas \& pjen6afes \\
\hline & $\therefore$ & $\cdots$ & \begin{tabular}{r}
$\square$ \\
\hdashline \\
\end{tabular} & a & & & & әuอ! 6 КН \\
\hline & & ( & 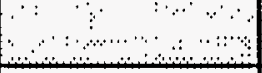 & $\because$ & & & & 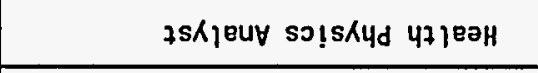 \\
\hline 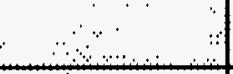 & $\therefore$ & $\because \quad \because \quad \therefore$ & $\therefore$ & $\ldots$ & & & & yos/Jauuejd/7ş 7soj \\
\hline$\therefore$ & \%: & - 3 & - & $\because \quad \pi$ & & & & jeut wə7S/s Jəznduro \\
\hline 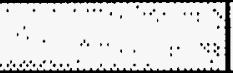 & औn. & S: : : : & +e & : & & & & S.07oəadsu! әsue! jduoJ \\
\hline : $: \quad: \quad$ & (1) & $\begin{array}{l}\text { की } \\
\text { us }\end{array}$ & ४थे & म & & & & 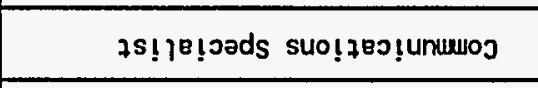 \\
\hline ' $\cdots \cdots$ & ४िमे & man & W. & खे & & & & J07!pne/zuezunosory \\
\hline \multicolumn{9}{|c|}{ : } \\
\hline 0.5 & 0.5 & 0.5 & $0^{\circ} \mathrm{s}$ & $0^{\circ} \mathrm{s}$ & 0.5 & 0.5 & $0 \cdot 5$ & 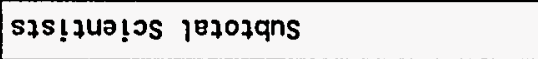 \\
\hline 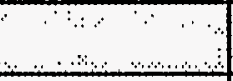 & 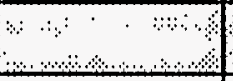 & $8 \cdots$ & (2) : : & $\therefore \cdots \cdots$ & 0.1 & 0.1 & 10.1 & دәч70 \\
\hline & . & $\because$ & 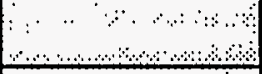 & $\because 2$ & & & & $75 !$ \\
\hline 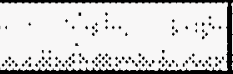 & अ: & 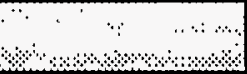 & 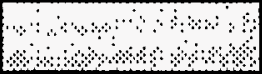 & अ & 0.7 & 0.7 & 0.7 & 47)Еан s7s!O!sKyd \\
\hline (1) & मेकिके & ४ें। & मै. & 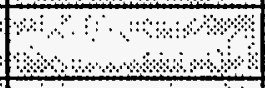 & & & & sue!ว! дешәчұеW \\
\hline & \% & 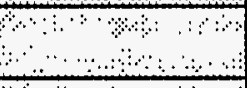 & 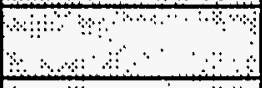 & 40 & & & & af!7 \\
\hline$\because$ & $\begin{array}{l}\because \cdots \\
\because \because \cdots \\
\end{array}$ & & $\therefore$. & & & & & 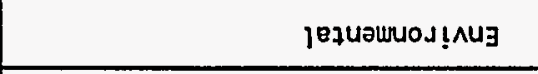 \\
\hline & $\therefore$ & & & & & & & s7s เุฒวบว \\
\hline & & & & & & & & SLSIINaIos \\
\hline 1002 & 0002 & 6661 & $866 !$ & 2661 & 9661 & 5666 & 7661 & ᄉsoбəzeว qor \\
\hline \multicolumn{9}{|c|}{ 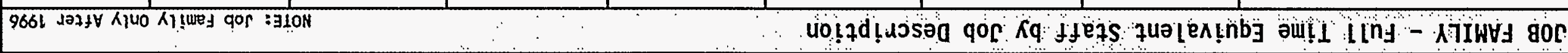 } \\
\hline $\begin{array}{l}\text { †6/IE/ } \\
\text { ueld ue }\end{array}$ & 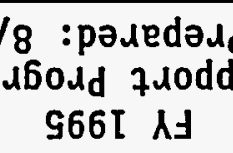 & $\begin{array}{l}\text { d әqеQ } \\
\text { lns } ә 7 ! s\end{array}$ & & $\begin{array}{l}\text { ssəup } \\
\text { אuedwoj }\end{array}$ & $\begin{array}{l}\varepsilon^{\cdot} Z^{\bullet} L^{\cdot} \\
\text { pasedald } \\
\text { puofueh }\end{array}$ & 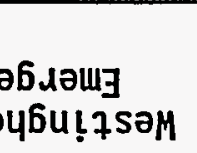 & & 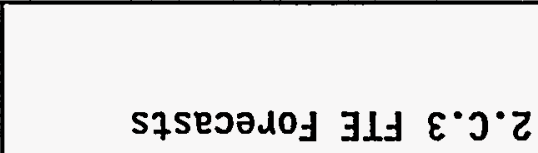 \\
\hline
\end{tabular}




\begin{tabular}{|c|c|c|c|c|c|c|c|c|}
\hline 2.C.3 FTE Forecasts & \multicolumn{5}{|c|}{$\begin{array}{c}\text { Westinghouse Hanford Company } \\
\text { Emergency Preparedness } \\
6.7 .2 .3\end{array}$} & \multicolumn{3}{|c|}{$\begin{array}{c}\text { FY } 1995 \\
\text { Site Support Program Plan } \\
\text { Date Prepared: } 8 / 31 / 94\end{array}$} \\
\hline \multicolumn{9}{|c|}{ JOB FAMILY - Full Time Equivalent staff by Job Description } \\
\hline Job category & 1994 & 1995 & 1996 & 1997 & 1998 & 1999 & 2000 & 2001 \\
\hline \multicolumn{9}{|l|}{ GEN ADM/SECRETARY/CLERK } \\
\hline \multicolumn{9}{|l|}{ Admin Assistants } \\
\hline office Clerks (Gen) & 1.0 & 1.0 & 1.0 & $\therefore \quad \therefore$ & & 0 & & \\
\hline Office Clerks (Special) & & & & $\therefore \cdots$ & 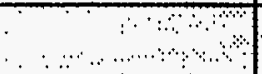 & a: & $\therefore \quad \therefore \quad \therefore \quad \because$ & $\because \cdots$ \\
\hline Secretaries & 4.0 & 3.0 & 3.0 & का & बिका & मेनि & : खो & a $\quad \cdots \cdots$ \\
\hline Typist/Word Process & & & & Q & $\because \quad \therefore \quad 1$ & 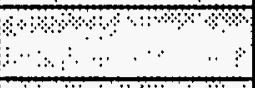 & \begin{tabular}{|lll} 
& \\
$\cdots$ & $\cdots$
\end{tabular} & 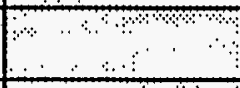 \\
\hline \multicolumn{9}{|l|}{ Other } \\
\hline Subtotal Gen Adm/Secretary/Clerk & 5.0 & 4.0 & 4.0 & 4.0 & 4.0 & 4.0 & 4.0 & 4.0 \\
\hline \multicolumn{9}{|l|}{ TECHNICHANS } \\
\hline Computer Oper/Coder & & & & में & कि & + & & (1) \\
\hline Engrs/Tech & & & & अैखि & $\begin{array}{c}p_{1} \\
\cdots \cdots \\
\end{array}$ & $\begin{array}{lll}+4 & \vdots \\
\end{array}$ & (n. & 8 \\
\hline Envir. Sci Technicians & & & & मै। & \% & \&. & 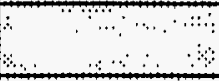 & $\begin{array}{c}1 \\
: \quad: \\
\end{array}$ \\
\hline Heal th Phys. Technic. & & & & Pै: & $\begin{array}{r}0+1 \\
\\
\end{array}$ & , & 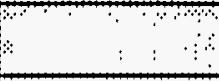 & \\
\hline Indus. Saf/Heal th Tech & & & & an & $\begin{array}{r} \\
\therefore \quad \therefore \quad \cdots \\
\end{array}$ & 6 & & $\because:$ \\
\hline Instru/Control rech & & & & \begin{tabular}{llll} 
& 0 & \\
\hdashline & $\cdots$
\end{tabular} & $\therefore$ & & $\because$ & \\
\hline Other & & & & \begin{tabular}{|lllll} 
& & & \\
& & & \\
\end{tabular} & 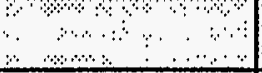 & $\therefore$ & & \\
\hline Subtotal Technicians & 0.0 & 0.0 & 0.0 & 0.0 & 0.0 & 0.0 & 0.0 & 0.0 \\
\hline
\end{tabular}




\begin{tabular}{|c|c|c|c|c|c|c|c|c|}
\hline 2.C.3 FTE Forecasts & \multicolumn{4}{|c|}{$\begin{array}{c}\text { Hestinghouse Hanford Company } \\
\text { Emergency Preparedness } \\
6.7 .2 .3\end{array}$} & & \multicolumn{3}{|c|}{$\begin{array}{c}\text { FY } 1995 \\
\text { Site Support Program Plan } \\
\text { Date Prepared: } 8 / 31 / 94\end{array}$} \\
\hline \multicolumn{9}{|c|}{ JOB FAMILY - FuII Time Equivalent Staff by Job Description } \\
\hline Job category & 1994 & 1995 & 1996 & 1997 & 1998 & 1999 & 2000 & 2001 \\
\hline \multicolumn{9}{|l|}{ CRAFTS } \\
\hline \multicolumn{9}{|l|}{ Electricians } \\
\hline \multicolumn{9}{|l|}{ other } \\
\hline Instrument Specialist & & & & 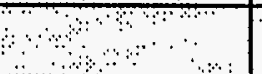 & r & ma & $\therefore$ & $\therefore: \cdots$ \\
\hline Locksmith Safemaster & & & & अै। & 2 & ڤ.: & on & N \\
\hline Subtotal Crafts & 0.0 & 0.0 & 0.0 & 0.0 & 0.0 & 0.0 & 0.0 & 0.0 \\
\hline \multicolumn{9}{|c|}{ LABOR \& GEN WORKERS U } \\
\hline Firefighters & & & & मे० & $\therefore$ & & $\therefore$ & : \\
\hline Security Guards & & & & अ०: & , & \begin{tabular}{|lll}
$y$ & \\
1 & & 1 \\
\end{tabular} & ..? & \% \\
\hline other & & & & मै। & $\because \quad \cdots$ & 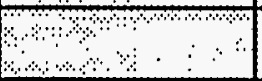 & & $2 \%$ \\
\hline Paramedics & & & & \%ै। & - & $\begin{array}{|lll|}\text { +e } & & \\
\end{array}$ & 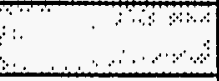 & $\begin{array}{ll} \\
\therefore \quad \cdots \quad \\
\end{array}$ \\
\hline Subtotal Labor \& Gen Workers & 0.0 & 0.0 & 0.0 & 0.0 & 0.0 & 0.0 & 0.0 & 0.0 \\
\hline Total FTEs 6.7 .2 .3 & 40.0 & 38.0 & 38.0 & 38.0 & 38.0 & 38.0 & 38.0 & 38.0 \\
\hline
\end{tabular}


$\vdots$

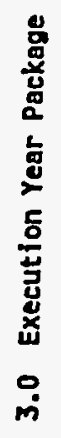




\begin{tabular}{|c|c|c|c|c|c|}
\hline \multirow{6}{*}{$\begin{array}{l}\text { Work } \\
\text { Breakdown } \\
\text { Structure } \\
\text { Dictionary }\end{array}$} & \multirow{6}{*}{\multicolumn{3}{|c|}{$\begin{array}{c}\text { Westinghouse Hanford Company } \\
\text { EMERGENCY PREPARDNESS } \\
\text { Part I - Summary } \\
\text { (Dollars In 000's) }\end{array}$}} & & \\
\hline & & & & \multirow{7}{*}{$\begin{array}{l}\text { FY } 1995 \text { SSPP } \\
\text { Rev. \# } 0\end{array}$} & \multirow{2}{*}{ Fundln! } \\
\hline & & & & & \\
\hline & & & & & \multirow{2}{*}{ OST } \\
\hline & & & & & \\
\hline & & & & & MGT PRO \\
\hline \multirow[t]{2}{*}{ Cost Account } & \multirow{2}{*}{\multicolumn{3}{|c|}{$\begin{array}{l}\text { Cost Account TIIle } \\
\text { FMERGENCY PREPAREDNESS }\end{array}$}} & & POOL \\
\hline & & & & & DIREGT \\
\hline SMS WBS $\quad 6.7 .2 .3$ & \multicolumn{2}{|c|}{ SMS TIIIO } & EMERGENCY PREPARED NESS & \multirow{2}{*}{\multicolumn{2}{|c|}{$\begin{array}{c}\text { Annualized Rate } \\
\text { (For Organizallonal Overhead and } \\
\text { Rated Service Pool Use Only) }\end{array}$}} \\
\hline \multicolumn{4}{|l|}{ GAM Revlew/Approval } & & \\
\hline \multicolumn{2}{|l|}{ SMS Program Manager Revlew/Approval } & \multicolumn{2}{|r|}{ Date } & FY 1994 Rate & FY 1995 Rale Reques! \\
\hline Financlal Manager Rovlew/Approval & L.C. Brandt & \multicolumn{2}{|r|}{ Date } & & \multirow{2}{*}{ FY 1995 Approved Rate } \\
\hline Responslble Analyst & P. S. Ripley & & Date & FY 1995 Targel Rate & \\
\hline \multirow[t]{2}{*}{ OSBRB Revlow/Approval } & B. R. Thomas & \multicolumn{2}{|r|}{ Date } & & ( \\
\hline & \multicolumn{2}{|c|}{ FY1994 } & \multicolumn{3}{|c|}{ FY 1995} \\
\hline FULL-TIME EQUIVALENTS (FTES) & Budget & $\begin{array}{c}\text { Fiscal Year Spending } \\
\text { Forecasl (FYSF) }\end{array}$ & Target & Request & $\begin{array}{l}\text { Approved } \\
\text { Basellne }\end{array}$ \\
\hline Organizallonal - Exompl & 32.5 & 32.5 & & 33.0 & \\
\hline Organizatlonal - Nonexempt & 7.0 & 7.0 & & 5.0 & \\
\hline \multicolumn{6}{|l|}{ Organlzatlonal - Bargalning } \\
\hline Total Organizallonal FTEs & 39.5 & 39.5 & & 38.0 & \\
\hline Support FTEs & 5.5 & 5.5 & & 4.0 & \\
\hline TOTALFTES & 45.0 & 45.0 & & 42.0 & \\
\hline \multicolumn{6}{|l|}{ COST ELEMENTS } \\
\hline . Labor - Regular & 2,675 & 2,634 & & 2,553 & \\
\hline Labor - Overtlme & 28 & 46 & & 35 & \\
\hline 0 Tolal Labor & 2,704 & 2,680 & & 2,588 & \\
\hline 1 Matellals & 274 & 232 & & 270 & \\
\hline 2 Purchased Services & 358 & 300 & & 320 & \\
\hline 3 Other Hanlord & 1,030 & 1,030 & & 507 & \\
\hline 4 Slle Services & 313 & 370 & & 338 & \\
\hline 5 Inlernal Charges & 54 & 95 & & 80 & \\
\hline 6 IAM Support & 459 & 503 & & 534 & \\
\hline 7 Overheads & 219 & 200 & & 163 & \\
\hline 8 Revenue & & & & & \\
\hline TOTALDOLLARS & 5,410 & 5.410 & & 4.800 & \\
\hline
\end{tabular}




\begin{tabular}{|l|c|c|}
\hline $\begin{array}{c}1 \mathrm{Ml} \\
\text { COST ACCOUNT }\end{array}$ & \multicolumn{2}{|c|}{} \\
\hline $\begin{array}{l}\text { Work Breakdown } \\
\begin{array}{l}\text { Structure } \\
\text { Dictionary }\end{array}\end{array}$ & Westinghouse Hanford Company & FY 1995 SSPP \\
\cline { 2 - 3 } & Part II - Elemenent Definition & Revision \# 0 \\
\hline
\end{tabular}

\begin{tabular}{|l|l|}
\hline WBS ELEMENT CODE: $1 M$ MDOL & TITLE: EMERGENCY PREPAREDNESS \\
\hline ELEMENT TASKDESCRIPTION & COST CONTENT - \\
The Emergency Preparedness (EP) budget source is SWS - Site Wide Support (formerly Common Support Pool) and is approved by the WHC Overhead Review \\
Board and the RL Budget Review Board. The elements of cost include labor, material, purchased services, other contractor services, site services, site support
\end{tabular}
and BCSR services.

\section{TECHNICAL CONTENT -}

EP provides emergency management for WHC and emergency management support for RL and the other Hanford Site contractors in order to maintain compliance with the DOE 5500 series orders. EP also provides occurrence reporting management for WHC and the Hanford Site to maintain compliance with DOE Order 5000.3B.

\section{OBJECTIVES -}

The 1995 objectives of the Westinghouse Hanford Company (WHC) Emergency Preparedness Program are to maintain compliance with the DOE Orders for emergency preparedness and occurrence reporting and the Emergency Management Guidelines.

The program will continue to provide quality products and services and continue to enhance the Site Emergency Preparedness Program.

\section{ASSUMPTIONS/CONSTRAINTS -}

Facility status will not cause a change to Emergency Preparedness support during FY 1995.

No significant changes to DOE Orders and federal regulations are anticipated that will impact the Hanford Site beyond this plan.

\section{MILESTONES -}

There are 27 milestones for Emergency Preparedness (6 RL and 21 WHC milestones). The milestones are listed in the milestone description sheets and the milestone control log.

DELIVERABLES -

Emergency Preparedness deliverables are associated with each milestone and are described in the milestone description sheets. 
Cost Account $1 \mathrm{MDBOL}$

Part II - - lement Definition (continued)

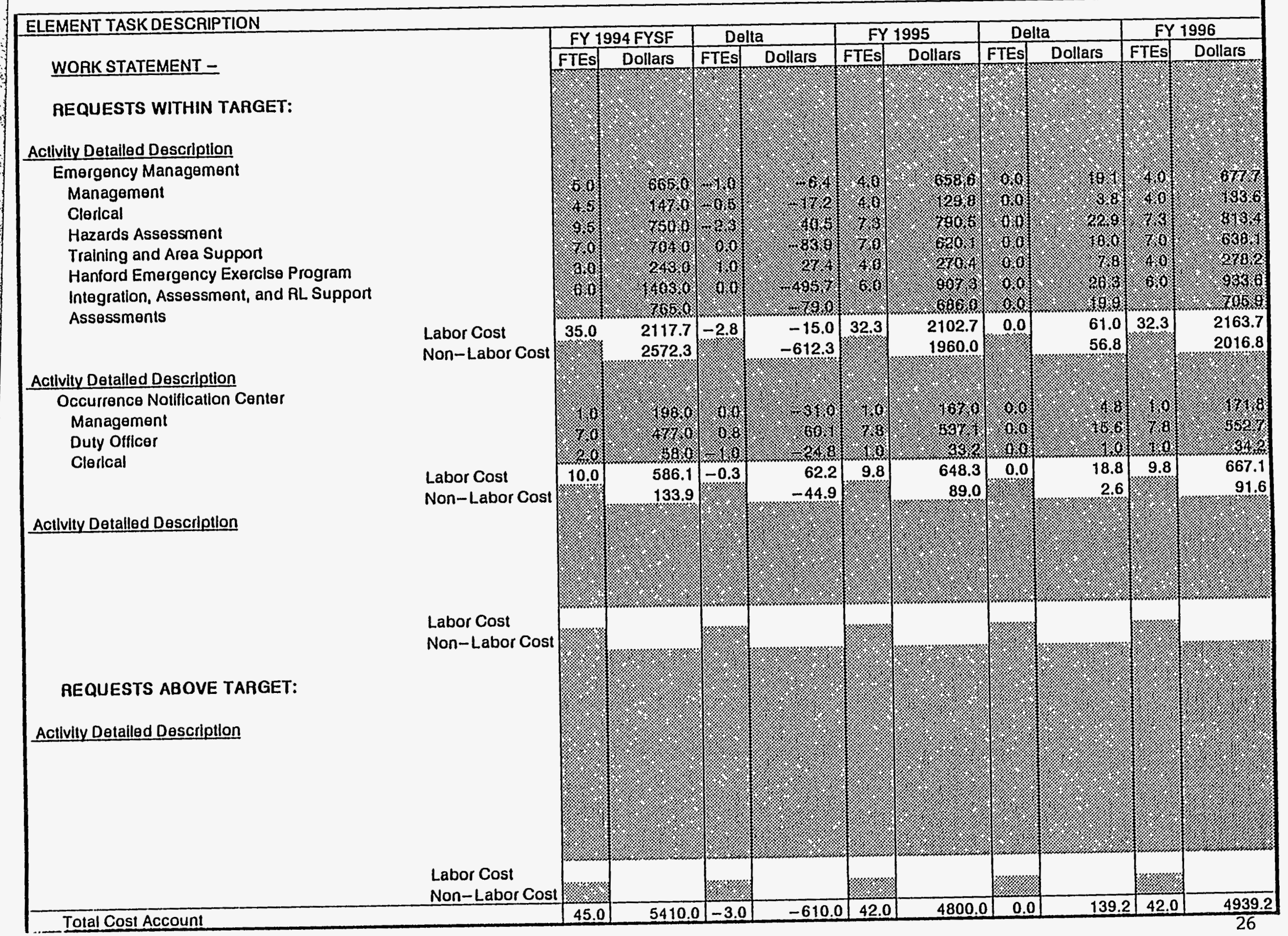


Westinghouse Hanford Company

MILESTONE DESCRIPTION SHEET

Title:

Implement Emergency Operations Center Project

\begin{tabular}{l|l|l}
\hline Assigned To: JW Tritz & CIN:
\end{tabular}

\begin{tabular}{|c|c|c|c|}
\hline WBS Designator: & \multicolumn{2}{|l|}{6.7 .2 .3} & $\begin{array}{l}\text { Due Date: } \\
1 / 31 / 95\end{array}$ \\
\hline Control Number: & \multicolumn{2}{|l|}{ HEP-95-001 } & Revision: 0 \\
\hline $\begin{array}{l}\text { Milestone Type: } \\
\square \text { DOE-HQ } \\
X \text { DOE-RL } \\
\square \text { CNTR }\end{array}$ & \begin{tabular}{|ll} 
& Division: \\
$\square$ & State \\
$\square$ & Federat \\
$X$ & DOE \\
$\square$ & RCRA \\
$\square$ & TPA\# \\
\end{tabular} & $\begin{array}{ll} & \text { DELIVERABLE: } \\
\square & \text { Report } \\
\square & \text { Letter } \\
\square & \text { Drawings } \\
X & \text { Other } \\
\text { (specify) } & \\
& \text { See description }\end{array}$ & $\begin{array}{ll} & \text { ADDRESS TO: } \\
\square & \text { DOE-HQ } \\
X & \text { DOE-RL } \\
\square & \text { Other } \\
\text { (specify) }\end{array}$ \\
\hline
\end{tabular}

\section{Milestone Description}

Complete Emergency Operations Center Integration Project.

Description of what constitutes completion of this milestone:

Completion of WHC Milestones $\begin{array}{r}\text { HEP-95-002 } \\ \text { HEP-95-003 } \\ \text { HEP-95-004 }\end{array}$

\begin{tabular}{|ll|ll|}
\hline Cost Account Manager & Date & $\begin{array}{l}\text { Program/Project Manager } \\
\text { JW Tritz }\end{array}$ & Date \\
\hline Program Element Manager & Date & $\begin{array}{l}\text { DOE Monitor } \\
\text { JB Hall }\end{array}$ & Date \\
\hline
\end{tabular}




\begin{tabular}{|c|c|c|c|}
\hline \multicolumn{3}{|c|}{$\begin{array}{l}\text { Title: } \\
\text { Complete Construction of Emergency Operations Center }\end{array}$} & $\begin{array}{l}\text { Date Prepared: } \\
08 / 31 / 94\end{array}$ \\
\hline Assigned To: & \multicolumn{2}{|l|}{ JW Tritz } & CIN: \\
\hline WBS Designator: & \multicolumn{2}{|l|}{6.7 .2 .3} & $\begin{array}{l}\text { Due Date: } \\
1 / 01 / 95\end{array}$ \\
\hline \multicolumn{3}{|c|}{ Control Number: HEP-95-002 } & Revision: 0 \\
\hline $\begin{array}{l}\text { Milestone Type: } \\
\square \text { DOE-HQ } \\
\square \text { DOE-RL } \\
X \quad \text { CNTR }\end{array}$ & $\begin{array}{ll} & \text { Division: } \\
\square & \text { State } \\
\square & \text { Federa } \\
X & \text { DOE } \\
\square & \text { RCRA } \\
\square & \text { TPA\# } \\
\end{array}$ & $\begin{array}{ll} & \text { DELIVERABLE: } \\
\square & \text { Report } \\
\square & \text { Letter } \\
\square & \text { Drawings } \\
\mathrm{X} & \text { Other } \\
\text { (specify) } & \\
& \\
\text { See description }\end{array}$ & $\begin{array}{ll} & \text { ADDRESS TO: } \\
\square & \text { DOE-HQ } \\
X & \text { DOE-RL } \\
\square & \text { Other } \\
& \text { (specify) }\end{array}$ \\
\hline
\end{tabular}

\section{Milestone Description}

Complete construction of the Emergency Operations Center (EOC).

Description of what constitutes completion of this milestone:

Construction of the new EOC is completed and ready to occupy.

\begin{tabular}{|ll|ll|}
\hline Cost Account Manager & Date & $\begin{array}{l}\text { Program/Project Manager } \\
\text { JW Tritz }\end{array}$ & Date \\
\hline Program El ement Manager & Date & $\begin{array}{l}\text { DOE Monitor } \\
\text { JB Hall }\end{array}$ & Date \\
\hline
\end{tabular}




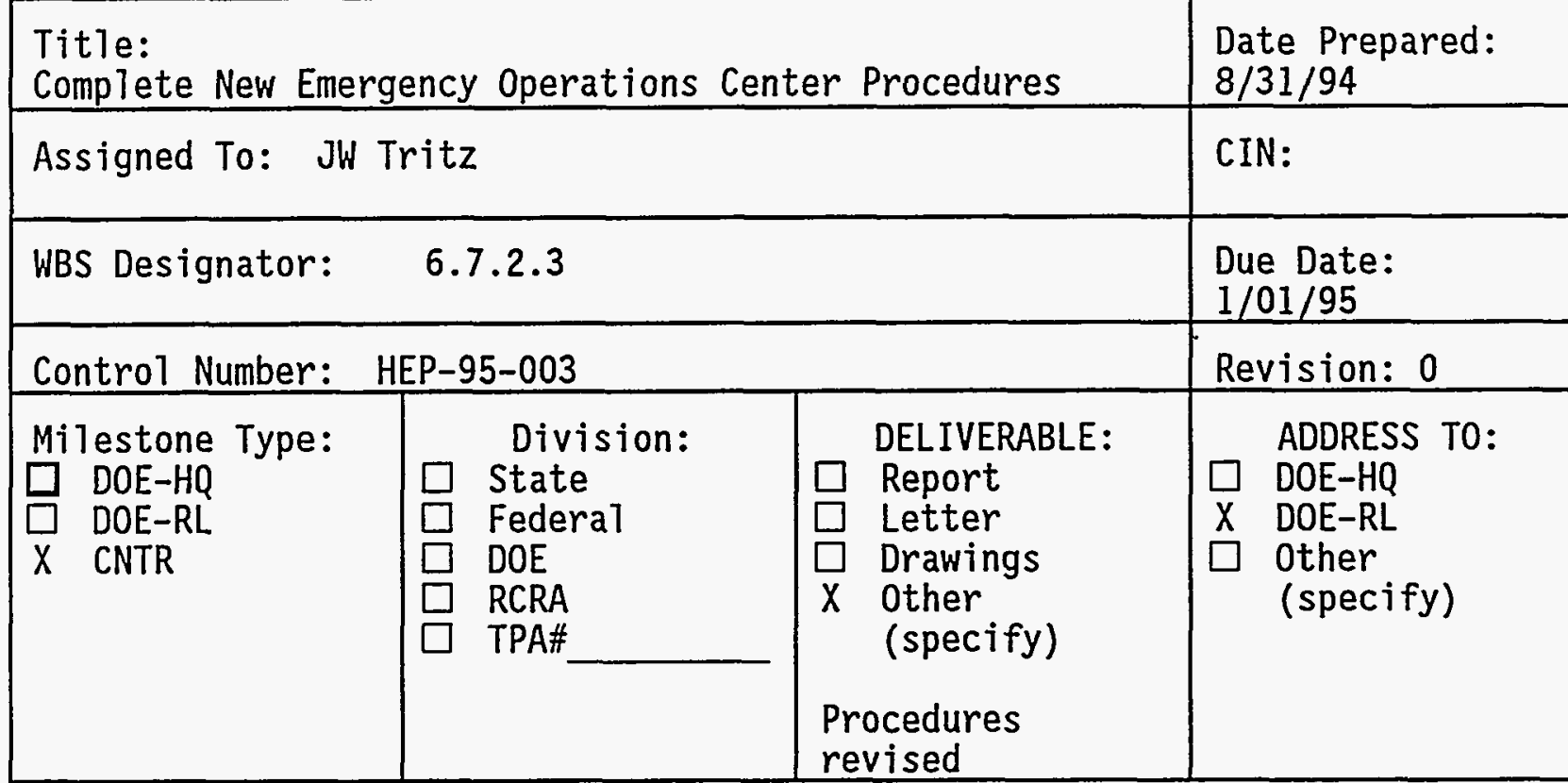

\section{Milestone Description}

Complete the procedures for operation of the new Emergency Operations Center.

Description of what constitutes completion of this milestone:

RLEP 2.0 revised and issued to reflect new positions in Emergency Operations Center.

\begin{tabular}{|ll|ll|}
\hline Cost Account Manager & Date & $\begin{array}{l}\text { Program/Project Manager } \\
\text { JW Tritz }\end{array}$ & Date \\
\hline Program Element Manager & Date & $\begin{array}{l}\text { DOE Monitor } \\
\text { JB Hall }\end{array}$ & Date \\
\hline
\end{tabular}


Title:

Complete Emergency Operations Center Personnel Training

Assigned To: JW Tritz

\begin{tabular}{|l|l|}
\hline WBS Designator: & \multicolumn{2}{c}{6.7 .2 .3} \\
\hline Control Number: & HEP-95-004 \\
\hline Milestone Type: & Division: \\
$\square$ DOE-HQ & $\square$ State \\
$\square$ DOE-RL & $\square$ Federal \\
$X$ CNTR & $\square$ DOE \\
& $\square$ RCRA \\
& $\square$ TPA\# \\
\hline
\end{tabular}

\section{Milestone Description}

Complete training of the Emergency Operations Center personnel using material developed using Performance Based Training techniques.

\section{Description of what constitutes completion of this milestone:}

Training scheduled and announced.

Scheduled Classes completed.

\begin{tabular}{|ll|ll|}
\hline Cost Account Manager & Date & $\begin{array}{l}\text { Program/Project Manager } \\
\text { JW Tritz }\end{array}$ & Date \\
\hline Program Element Manager & Date & $\begin{array}{l}\text { DOE Monitor } \\
\text { JB Hall }\end{array}$ & Date \\
\hline
\end{tabular}

Date Prepared:

$8 / 31 / 94$

CIN:

Due Date:

$1 / 31 / 95$

Revision: 0

ADDRESS T0:

DOE-HQ

$X$ DOE-RL

Other

(specify)

\section{Training}

completed

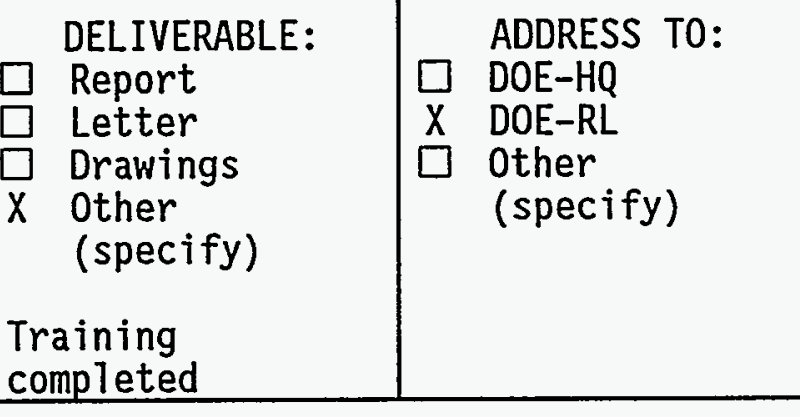




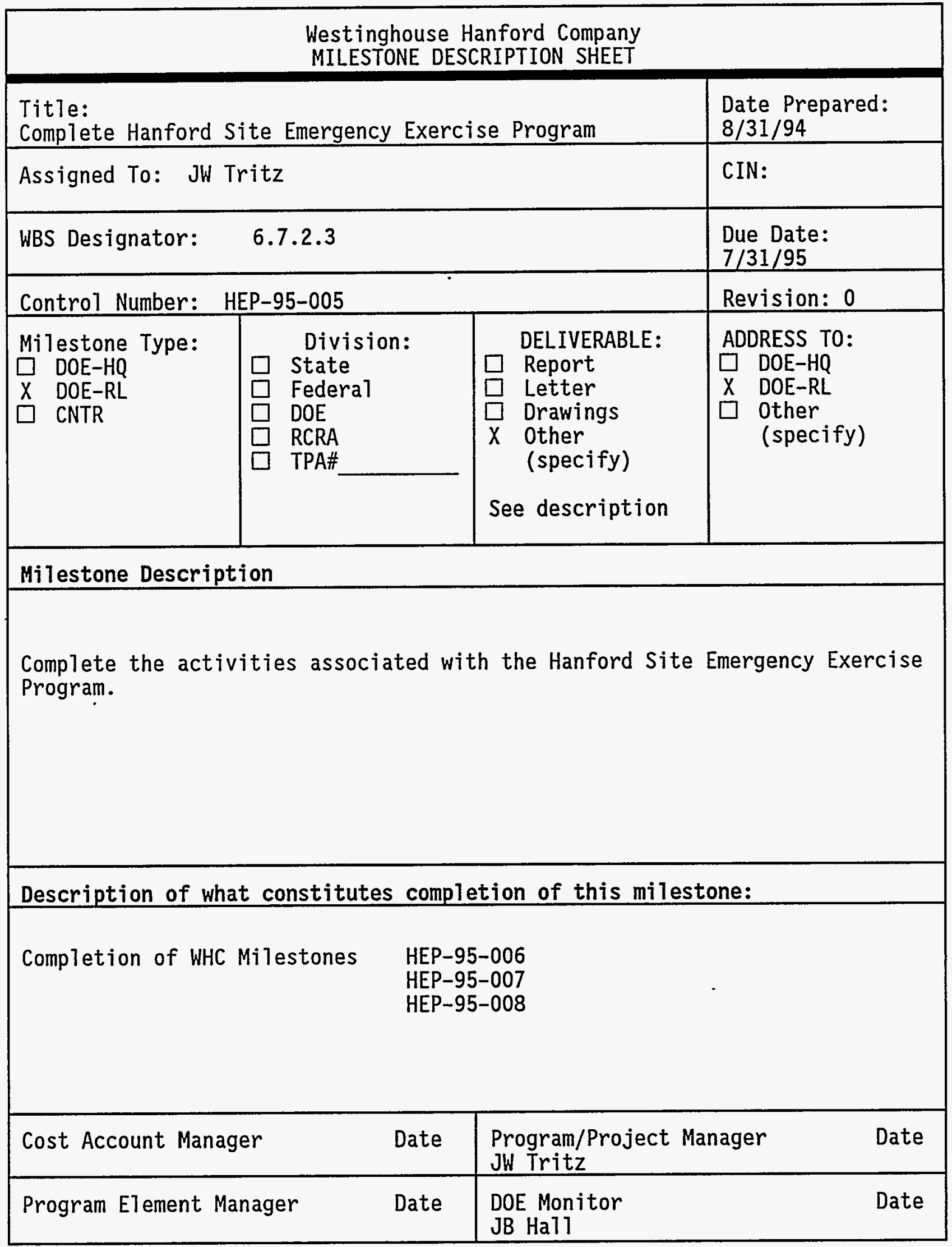




\begin{tabular}{|c|c|c|c|}
\hline \multicolumn{3}{|c|}{$\begin{array}{l}\text { Title: } \\
\text { Revise the Exercise Evaluation Process }\end{array}$} & $\begin{array}{l}\text { Date Prepared: } \\
8 / 31 / 94\end{array}$ \\
\hline \multicolumn{3}{|c|}{ Assigned To: JW Tritz } & CIN: \\
\hline WBS Designator: & 6.7 .2 .3 & & $\begin{array}{l}\text { Due Date: } \\
1 / 31 / 95 \\
\end{array}$ \\
\hline \multicolumn{3}{|c|}{ Control Number: HEP-95-006 } & Revision: 0 \\
\hline $\begin{array}{l}\text { MiTestone Type: } \\
\square \text { DOE-HQ } \\
\square \text { DOE-RL } \\
\square \text { CNTR }\end{array}$ & $\begin{array}{ll} & \text { Division: } \\
\square & \text { State } \\
\square & \text { Federa1 } \\
\square & \text { DOE } \\
\square & \text { RCRA } \\
\square & \text { TPA\# } \\
\end{array}$ & $\begin{array}{ll} & \text { DELIVERABLE: } \\
\square & \text { Report } \\
\square & \text { Letter } \\
\square & \text { Drawings } \\
X & \text { Other } \\
\text { (specify) } & \\
\text { Procedure revised }\end{array}$ & $\begin{array}{ll} & \text { ADDRESS T0: } \\
\square & \text { DOE-HQ } \\
X & \text { DOE-RL } \\
\square & \text { Other } \\
\text { (specify) }\end{array}$ \\
\hline
\end{tabular}

Revise the exercise evaluation procedure.

\section{Description of what constitutes completion of this milestone:}

Procedure G4.04 in WHC CM 4-43 revised and issued.

\begin{tabular}{|ll|lc|}
\hline Cost Account Manager & Date & $\begin{array}{l}\text { Program/Project Manager } \\
\text { JW Tritz }\end{array}$ & Date \\
\hline Program Element Manager & Date & $\begin{array}{l}\text { DOE Monitor } \\
\text { JB Hall }\end{array}$ & Date \\
\hline
\end{tabular}




\section{Title:}

Track Exercise Participation

Date Prepared:

Assigned To: JW Tritz

$8 / 31 / 94$

\begin{tabular}{|c|c|c|c|}
\hline WBS Designator: & \multicolumn{2}{|l|}{6.7 .2 .3} & $\begin{array}{l}\text { Due Date: } \\
3 / 31 / 95\end{array}$ \\
\hline \multicolumn{3}{|c|}{ Control Number: HEP-95-007 } & Revision: 0 \\
\hline $\begin{array}{l}\text { Milestone Type: } \\
\square \text { DOE-HQ } \\
\square \text { DOE-RL } \\
\text { X CNTR }\end{array}$ & $\begin{array}{ll} & \text { Division: } \\
\square & \text { State } \\
\square & \text { Federal } \\
\square & \text { DOE } \\
\square & \text { RCRA } \\
\square & \text { TPA\# }\end{array}$ & $\begin{array}{ll} & \text { DELIVERABLE: } \\
\square & \text { Report } \\
\square & \text { Letter } \\
\square & \text { Drawings } \\
X & \text { Other } \\
\text { (specify) } & \\
& \\
\text { Database } \\
\text { completed }\end{array}$ & $\begin{array}{ll} & \text { ADDRESS TO: } \\
\square & \text { DOE-HQ } \\
X & \text { DOE-RL } \\
\square & \text { Other } \\
& \text { (specify) }\end{array}$ \\
\hline
\end{tabular}

Milestone Description

Develop a method to track exercise participation by Emergency Response Organization members.

Description of what constitutes completion of this milestone:

Database developed.

\begin{tabular}{|ll|ll|}
\hline Cost Account Manager & Date & $\begin{array}{l}\text { Program/Project Manager } \\
\text { JW Tritz }\end{array}$ & Date \\
\hline Program Element Manager & Date & $\begin{array}{l}\text { DOE Monitor } \\
\text { JB Hall }\end{array}$ & Date \\
\hline
\end{tabular}




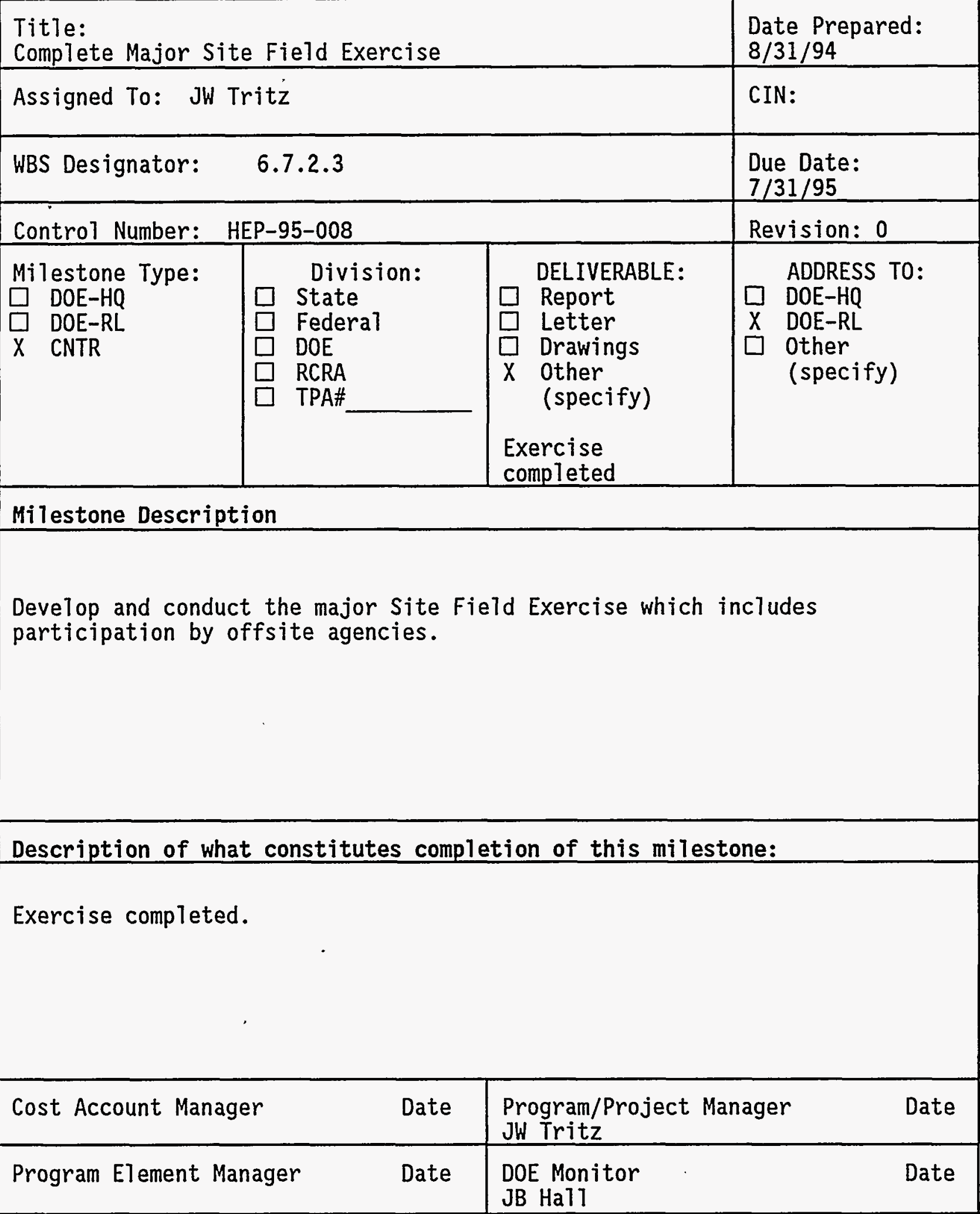




\begin{tabular}{|c|c|c|c|c|c|}
\hline \multicolumn{4}{|c|}{$\begin{array}{l}\text { Title: } \\
\text { Improve Occurrence Reporting System }\end{array}$} & $\begin{array}{l}\text { Date } \\
8 / 31 /\end{array}$ & \\
\hline \multicolumn{4}{|c|}{ Assigned To: JW Tritz } & CIN: & \\
\hline WBS Designator: & \multicolumn{3}{|l|}{6.7 .2 .3} & \multicolumn{2}{|c|}{$\begin{array}{l}\text { Due Date: } \\
9 / 30 / 95\end{array}$} \\
\hline \multicolumn{4}{|c|}{ Control Number: HEP-95-009 } & \multicolumn{2}{|c|}{ Revision: 0} \\
\hline $\begin{array}{l}\text { Milestone Type: } \\
\square \text { DOE-HQ } \\
X \text { DOE-RL } \\
\square \text { CNTR }\end{array}$ & \multicolumn{2}{|c|}{$\begin{array}{ll} & \text { Division: } \\
\square & \text { State } \\
\square & \text { Federat } \\
\square & \text { DOE } \\
\square & \text { RCRA } \\
\square & \text { TPA\# } \\
\end{array}$} & $\begin{array}{ll} & \text { DELIVERABLE: } \\
\square & \text { Report } \\
\square & \text { Letter } \\
\square & \text { Drawings } \\
X & \text { Other } \\
& \text { (specify) } \\
\text { See description }\end{array}$ & \multicolumn{2}{|c|}{$\begin{array}{ll} & \text { ADDRESS TO: } \\
\square & \text { DOE-HQ } \\
X & \text { DOE-RL } \\
\square & \text { Other } \\
\text { (specify) }\end{array}$} \\
\hline \multicolumn{6}{|c|}{ Milestone Description } \\
\hline \multicolumn{6}{|c|}{ Improve the Occurrence Reporting System. } \\
\hline \multicolumn{6}{|c|}{ Description of what constitutes completion of this milestone: } \\
\hline \multicolumn{4}{|c|}{$\begin{array}{cl}\text { Completion of WHC Milestones } & \text { HEP-95-010 } \\
& \text { HEP-95-011 } \\
& \text { HEP-95-012 } \\
& \text { HEP-95-013 } \\
& \text { HEP-95-014 }\end{array}$} & & \\
\hline \multicolumn{2}{|c|}{ Cost Account Manager } & Date & \multicolumn{2}{|c|}{$\begin{array}{l}\text { Program/Project Manager } \\
\text { JW Tritz }\end{array}$} & Date \\
\hline \multicolumn{3}{|c|}{ Program Element Manager Date } & \multicolumn{3}{|c|}{$\begin{array}{l}\text { DOE Monitor } \\
\text { JB Hall }\end{array}$} \\
\hline
\end{tabular}




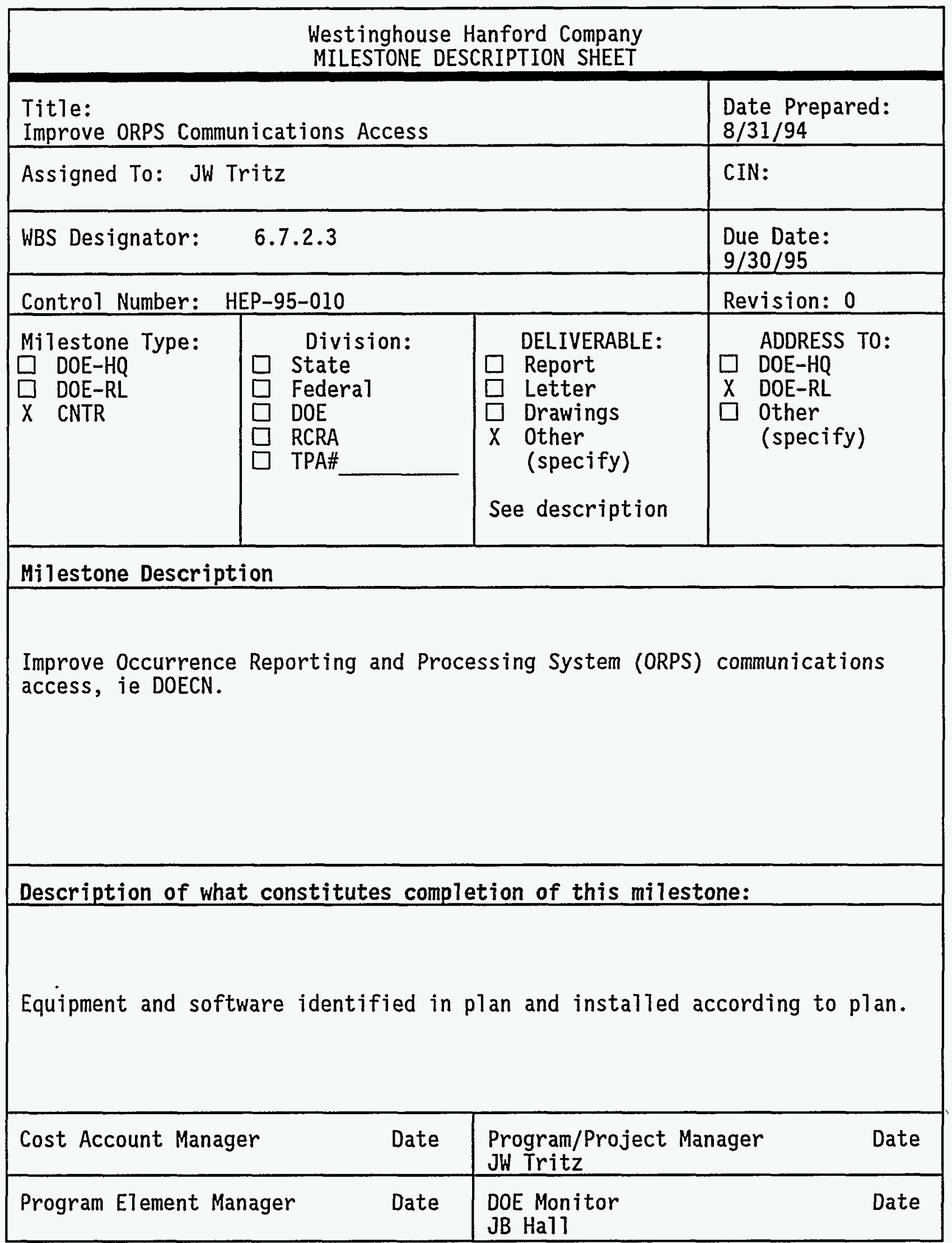




\begin{tabular}{|c|c|c|c|}
\hline \multicolumn{4}{|c|}{$\begin{array}{l}\text { Westinghouse Hanford Company } \\
\text { MILESTONE DESCRIPTION SHEET }\end{array}$} \\
\hline \multicolumn{3}{|c|}{$\begin{array}{l}\text { Title: } \\
\text { Implement Procedure Change Based Upon 5000.3B Change }\end{array}$} & $\begin{array}{l}\text { Date Prepared: } \\
8 / 31 / 94\end{array}$ \\
\hline \multicolumn{3}{|c|}{ Assigned To: JW Tritz } & CIN: \\
\hline WBS Designator: & \multicolumn{2}{|l|}{6.7 .2 .3} & $\begin{array}{l}\text { Due Date: } \\
90 \text { days from } \\
\text { issuance of } \\
\text { changes to Order }\end{array}$ \\
\hline \multicolumn{3}{|c|}{ Control Number: HEP-95-011 } & Revision: 0 \\
\hline $\begin{array}{l}\text { Milestone Type: } \\
\square \text { DOE-HQ } \\
\square \text { DOE-RL } \\
\text { X CNTR }\end{array}$ & $\begin{array}{ll} & \text { Division: } \\
\square & \text { State } \\
\square & \text { Federal } \\
\square & \text { DOE } \\
\square & \text { RCRA } \\
\square & \text { TPA\# } \\
\end{array}$ & $\begin{array}{ll} & \text { DELIVERABLE: } \\
\square & \text { Report } \\
\square & \text { Letter } \\
\square & \text { Drawings } \\
\mathrm{X} & \text { Other } \\
\text { (specify) } & \\
\text { Procedure revised }\end{array}$ & $\begin{array}{ll} & \text { ADDRESS TO: } \\
& \text { DOE-HQ } \\
X & \text { DOE-RL } \\
\square & \text { other } \\
& \text { (specify) }\end{array}$ \\
\hline \multicolumn{4}{|c|}{ Milestone Description } \\
\hline \multicolumn{4}{|c|}{$\begin{array}{l}\text { Implement Company level procedure changes necessary with changes initiated } \\
\text { against DOE Order } 5000.3 \mathrm{~B} \text {, "Occurrence Reporting and Processing of } \\
\text { Operations Information." }\end{array}$} \\
\hline \multicolumn{4}{|c|}{ Description of what constitutes completion of this milestone: } \\
\hline \multicolumn{4}{|c|}{ Management Requirements and Procedures 5.14 revised. } \\
\hline Cost Account Manage & Date & $\begin{array}{l}\text { Program/Project Ma } \\
\text { JW Tritz }\end{array}$ & nager \\
\hline Program Element Man & Date & $\begin{array}{l}\text { DOE Monitor } \\
\text { JB Hall }\end{array}$ & Date \\
\hline
\end{tabular}




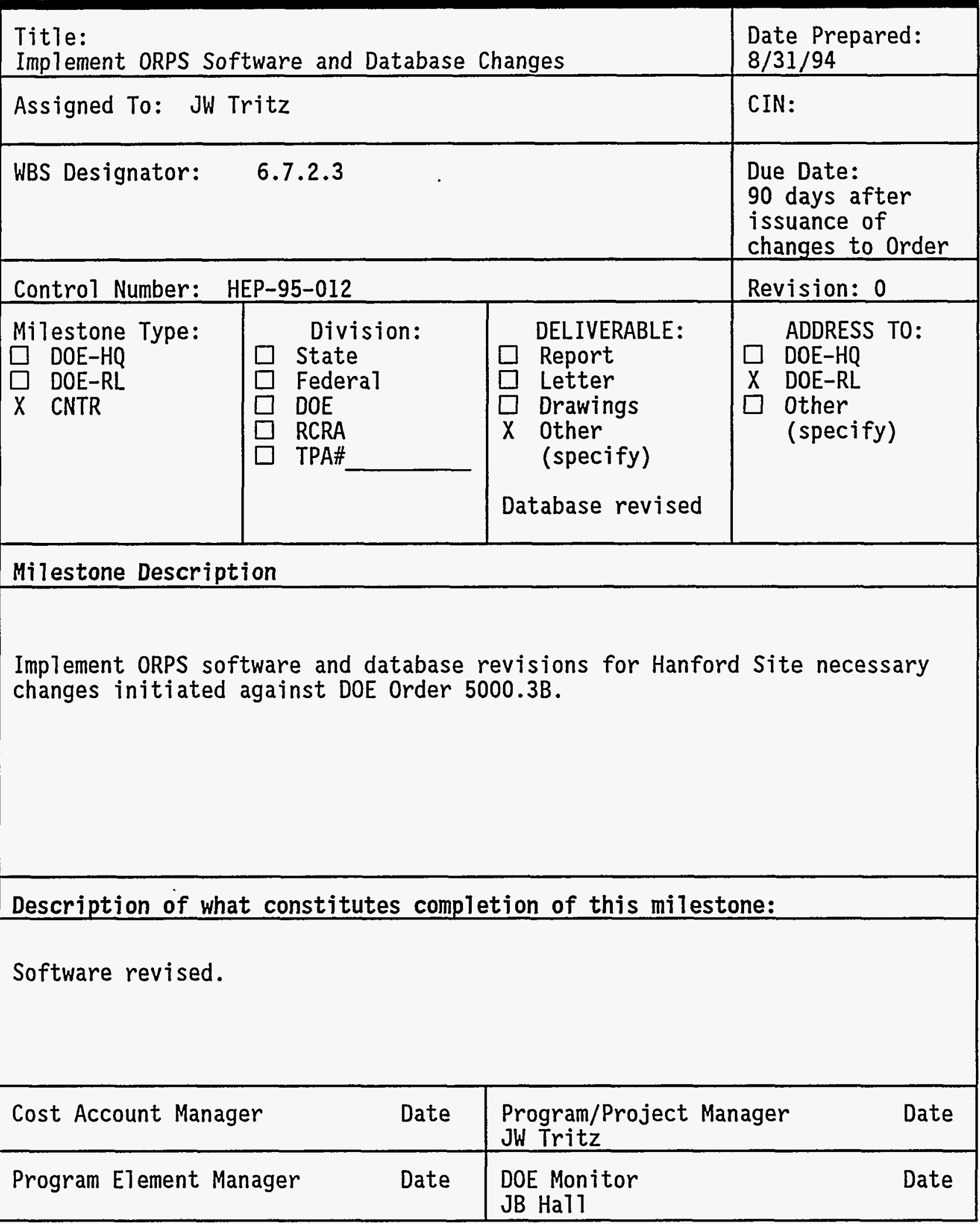




\begin{tabular}{|c|c|c|c|}
\hline \multicolumn{3}{|c|}{$\begin{array}{l}\text { Tit7e: } \\
\text { Revise QTRC Occurrence Reporting Training Program }\end{array}$} & $\begin{array}{l}\text { Date Prepared: } \\
8 / 31 / 94\end{array}$ \\
\hline \multicolumn{3}{|c|}{ Assigned To: JW Tritz } & CIN: \\
\hline WBS Designator: & \multicolumn{2}{|l|}{6.7 .2 .3} & $\begin{array}{l}\text { Due Date: } \\
90 \text { days after } \\
\text { issuance of } \\
\text { changes to Order }\end{array}$ \\
\hline \multicolumn{3}{|c|}{ Control Number: HEP-95-013 } & Revision: 0 \\
\hline $\begin{array}{l}\text { Milestone Type: } \\
\square \text { DOE-HQ } \\
\square \text { DOE-RL } \\
\text { X CNTR }\end{array}$ & $\begin{array}{ll} & \text { Division: } \\
\square & \text { State } \\
\square & \text { Federal } \\
\square & \text { DOE } \\
\square & \text { RCRA } \\
\square & \text { TPA\# }\end{array}$ & $\begin{array}{ll} & \text { DELIVERABLE: } \\
& \text { Report } \\
\square & \text { Letter } \\
\square & \text { Drawings } \\
\mathrm{X} & \text { Other } \\
& \text { (specify) } \\
& \\
\text { Lesson Plans } \\
\text { revised }\end{array}$ & $\begin{array}{ll} & \text { ADDRESS TO: } \\
\square & \text { DOE-HQ } \\
X & \text { DOE-RL } \\
\square & \text { 0ther } \\
& \text { (specify) }\end{array}$ \\
\hline
\end{tabular}

Revise QTRC training program changes necessary with changes initiated against DOE Order 5000.3B.

Description of what constitutes completion of this milestone:

Lesson Plans and visual aids revised and ready to use for training.

\begin{tabular}{|ll|lc|}
\hline Cost Account Manager & Date & $\begin{array}{l}\text { Program/Project Manager } \\
\text { JW Tritz }\end{array}$ & Date \\
\hline Program El ement Manager & Date & $\begin{array}{l}\text { DOE Monitor } \\
\text { JB Hall }\end{array}$ & Date \\
\hline
\end{tabular}




\begin{tabular}{|c|c|c|c|}
\hline \multicolumn{3}{|c|}{$\begin{array}{l}\text { Title: } \\
\text { Develop Assessment/Oversight P1an for Occurrence } \\
\text { Reporting }\end{array}$} & $\begin{array}{l}\text { Date Prepared: } \\
8 / 31 / 94\end{array}$ \\
\hline \multicolumn{3}{|c|}{ Assigned To: JW Tritz } & CIN: \\
\hline WBS Designator: & \multicolumn{2}{|l|}{6.7 .2 .3} & $\begin{array}{l}\text { Due Date: } \\
7 / 31 / 95\end{array}$ \\
\hline \multicolumn{3}{|c|}{ Control Number: HEP-95-014 } & Revision: 0 \\
\hline $\begin{array}{l}\text { Milestone Type: } \\
\square \text { DOE-HQ } \\
\square \text { DOE-RL } \\
\text { X CNTR }\end{array}$ & $\begin{array}{ll} & \text { Division: } \\
\square & \text { State } \\
\square & \text { Federat } \\
\square & \text { DOE } \\
\square & \text { RCRA } \\
\square & \text { TPA\# } \\
\end{array}$ & $\begin{array}{ll} & \text { DELIVERABLE: } \\
\square & \text { Report } \\
\square & \text { Letter } \\
\square & \text { Drawings } \\
\mathrm{X} & \text { Other } \\
& \text { (specify) } \\
& \\
\text { Plan issued }\end{array}$ & $\begin{array}{ll} & \text { ADDRESS TO: } \\
\square & \text { DOE-HQ } \\
X & \text { DOE-RL } \\
\square & \text { Other } \\
& \text { (specify) }\end{array}$ \\
\hline
\end{tabular}

Develop an Assessment/Oversight $\mathrm{pl}$ an for Occurrence Reporting.

Description of what constitutes completion of this milestone:

Plan completed and approved.

\begin{tabular}{|ll|lc|}
\hline Cost Account Manager & Date & $\begin{array}{l}\text { Program/Project Manager } \\
\text { JW Tritz }\end{array}$ & Date \\
\hline Program Element Manager & Date & $\begin{array}{l}\text { DOE Monitor } \\
\text { JB Hall }\end{array}$ & Date \\
\hline
\end{tabular}




\begin{tabular}{|c|c|c|c|c|c|}
\hline $\begin{array}{l}\text { Title: } \\
\text { Enhance Offsite }\end{array}$ & terface Pro & ram & & $\begin{array}{l}\text { Date } \\
8 / 31 \\
\end{array}$ & $\begin{array}{l}\text { e Prepared: } \\
1 / 94\end{array}$ \\
\hline Assigned To: JW & ritz & & & CIN: & \\
\hline WBS Designator: & 6.7 .2 .3 & & & $\begin{array}{l}\text { Due } \\
9 / 30 \\
\end{array}$ & $\begin{array}{l}\text { Date: } \\
0 / 95\end{array}$ \\
\hline Control Number: & EP-95-015 & & & Revi & ision: 0 \\
\hline $\begin{array}{l}\text { Milestone Type: } \\
\square \text { DOE-HQ } \\
X \text { DOE-RL } \\
\square \text { CNTR }\end{array}$ & \begin{tabular}{ll} 
& \multicolumn{1}{c}{ Divis } \\
$\square$ & State \\
$\square$ & Federa \\
$\square$ & DOE \\
$\square$ & RCRA \\
$\square$ & TPA\# \\
\end{tabular} & & $\begin{array}{ll} & \text { DELIVERABLE: } \\
\square & \text { Report } \\
\square & \text { Letter } \\
\square & \text { Drawings } \\
X & \text { Other } \\
\text { (specify) }\end{array}$ & $\begin{array}{ll} & A \\
\square & D \\
X & D \\
\square & 0 \\
& 1\end{array}$ & $\begin{array}{l}\text { ADDRESS TO: } \\
\text { DOE-HQ } \\
\text { DOE-RL } \\
\text { Dther } \\
\text { (specify) }\end{array}$ \\
\hline Milestone Descrip & ion & & & & \\
\hline Enhance the offsi & e interface & progra & & & \\
\hline Description of wh & $t$ constitut & s comp & tion of this mile & one: & \\
\hline Completion of WHC & Milestones & $\begin{array}{l}\text { HEP-C } \\
\text { HEP-C } \\
\text { HEP-C } \\
\text { HEP-C } \\
\text { HEP-S }\end{array}$ & $\begin{array}{l}.016 \\
.017 \\
018 \\
.019 \\
020\end{array}$ & & \\
\hline Cost Account Mana & & Date & $\begin{array}{l}\text { Program/Project } \\
\text { JW Tritz }\end{array}$ & nager & Date \\
\hline Program ETement $M$ & ager & Date & $\begin{array}{l}\text { DOE Monitor } \\
\text { JB Hall }\end{array}$ & & Date \\
\hline
\end{tabular}


Title:

Develop Electronic Notification Form

Date Prepared:

\begin{tabular}{|c|c|c|c|}
\hline \multirow{2}{*}{\multicolumn{3}{|c|}{ Assigned To: JW Tritz }} & \multirow{3}{*}{$\begin{array}{l}\text { CIN: } \\
\text { Due Date: } \\
1 / 31 / 95\end{array}$} \\
\hline & & & \\
\hline WBS Designator: & 6.7 .2 .3 & & \\
\hline \multicolumn{3}{|c|}{ Control Number: HEP-95-016 } & Revision: 0 \\
\hline 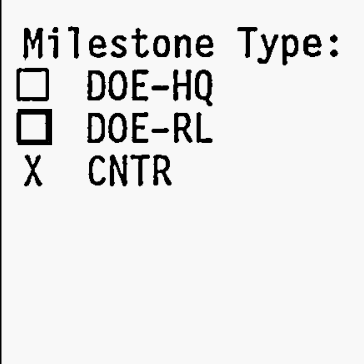 & $\begin{array}{l}\quad \text { Division: } \\
\square \text { State } \\
\square \text { Federal } \\
\square \text { DOE } \\
\square \text { RCRA } \\
\square \text { TPA\# } \\
\end{array}$ & $\begin{array}{ll} & \text { DELIVERABLE: } \\
\square & \text { Report } \\
\square & \text { Letter } \\
\square & \text { Drawings } \\
X & \text { Other } \\
\text { (specify) } & \\
\text { Form complete }\end{array}$ & $\begin{array}{ll} & \text { ADDRESS TO: } \\
\square & \text { DOE-HQ } \\
X & \text { DOE-RL } \\
\square & \text { Other } \\
\text { (specify) }\end{array}$ \\
\hline
\end{tabular}

Milestone Description

Develop an electronic notification form.

\section{Description of what constitutes completion of this milestone:}

Form completed and loaded onto a computer for use in Emergency Operation Center.

Personnel trained on use of the form.

\begin{tabular}{|ll|ll|}
\hline Cost Account Manager & Date & $\begin{array}{l}\text { Program/Project Manager } \\
\text { JW Tritz }\end{array}$ & Date \\
\hline Program Element Manager & Date & $\begin{array}{l}\text { DOE Monitor } \\
\text { JB Ha17 }\end{array}$ & Date \\
\hline
\end{tabular}


Title:

Fund Emergency Public Information Calendar

Date Prepared:

\begin{tabular}{|c|c|c|c|}
\hline \multicolumn{3}{|c|}{ Assigned To: JW Tritz } & CIN: \\
\hline WBS Designator: & 6.7 .2 .3 & & $\begin{array}{l}\text { Due Date: } \\
9 / 30 / 95\end{array}$ \\
\hline \multicolumn{3}{|c|}{ Control Number: HEP-95-017 } & Revision: 0 \\
\hline $\begin{array}{l}\text { Milestone Type: } \\
\square \text { DOE-HQ } \\
\square \text { DOE-RL } \\
X \text { CNTR }\end{array}$ & $\begin{array}{ll} & \text { Division: } \\
\square & \text { State } \\
\square & \text { Federal } \\
\square & \text { DOE } \\
\square & \text { RCRA } \\
\square & \text { TPA\# } \\
\end{array}$ & $\begin{array}{ll} & \text { DELIVERABLE: } \\
\square & \text { Report } \\
\square & \text { Letter } \\
\square & \text { Drawings } \\
\mathrm{X} & \text { Other } \\
\text { (specify) } & \\
\text { See description }\end{array}$ & $\begin{array}{ll} & \text { ADDRESS TO: } \\
\square & \text { DOE-HQ } \\
X & \text { DOE-RL } \\
\square & \text { Other } \\
\text { (specify) }\end{array}$ \\
\hline
\end{tabular}

Milestone Description

Coordinate with the Supply System and plume counties to develop the Emergency Pub7ic Information Calendar and pay the costs for the calendar in 1995 and odd years thereafter.

\section{Description of what constitutes completion of this milestone:}

1995 Calendar Costs paid

1996 Calendar format agreed to by Supply System, RL, Benton, Franklin, and Grant Counties for layout and printing

\begin{tabular}{|ll|lc|}
\hline Cost Account Manager & Date & $\begin{array}{l}\text { Program/Project Manager } \\
\text { JW Tritz }\end{array}$ & Date \\
\hline Program Element Manager & Date & $\begin{array}{l}\text { DOE Monitor } \\
\text { JB Hall }\end{array}$ & Date \\
\hline
\end{tabular}




\begin{tabular}{|c|c|c|c|}
\hline \multicolumn{3}{|c|}{$\begin{array}{l}\text { Title: } \\
\text { Provide Suggested Permanent EPZs, etc. }\end{array}$} & $\begin{array}{l}\text { Date Prepared: } \\
8 / 31 / 94\end{array}$ \\
\hline \multicolumn{3}{|c|}{ Assigned To: JW Tritz } & CIN: \\
\hline \multicolumn{3}{|c|}{ WBS Designator: $\quad 6.7 .2 .3$} & $\begin{array}{l}\text { Due Date: } \\
6 / 30 / 95\end{array}$ \\
\hline \multicolumn{3}{|c|}{ Control Number: HEP-95-018 } & Revision: 0 \\
\hline $\begin{array}{l}\text { Milestone Type: } \\
\square \text { DOE-HQ } \\
\square \text { DOE-RL } \\
X \text { CNTR }\end{array}$ & $\begin{array}{ll}\quad \text { Division: } \\
\square \text { State } \\
\square \text { Federal } \\
\square \text { DOE } \\
\square \text { RCRA } \\
\square \text { TPA\# }\end{array}$ & $\begin{array}{ll} & \text { DELIVERABLE: } \\
\square & \text { Report } \\
X & \text { Letter } \\
\square & \text { Drawings } \\
\square & \text { Other } \\
& \text { (specify) }\end{array}$ & $\begin{array}{ll} & \text { ADDRESS TO: } \\
\square & \text { DOE-HQ } \\
X & \text { DOE-RL } \\
\square & \text { Other } \\
\text { (specify) }\end{array}$ \\
\hline
\end{tabular}

\section{Milestone Description}

Provide suggested permanent EPZs, geopolitical boundaries and automatic protective action recommendations to the States and counties.

\section{Description of what constitutes completion of this milestone:}

Letter sent to Washington State Emergency Management Division containing permanent EPZs based on hazards assessments.

Letter sent to Benton, Franklin, and Grant County suggesting geopolitical boundaries and automatic protective actions.

\begin{tabular}{|ll|lc|}
\hline Cost Account Manager & Date & $\begin{array}{l}\text { Program/Project Manager } \\
\text { JW Tritz }\end{array}$ & Date \\
\hline Program Element Manager & Date & $\begin{array}{l}\text { DOE Monitor } \\
\text { JB Hall }\end{array}$ & Date \\
\hline
\end{tabular}




\begin{tabular}{|c|c|c|c|}
\hline \multicolumn{3}{|c|}{$\begin{array}{l}\text { Title: } \\
\text { Complete Engineering for Sirens }\end{array}$} & $\begin{array}{l}\text { Date Prepared: } \\
8 / 31 / 94\end{array}$ \\
\hline \multicolumn{3}{|c|}{ Assigned To: JW Tritz } & CIN: \\
\hline WBS Designator: & \multicolumn{2}{|l|}{6.7 .2 .3} & $\begin{array}{l}\text { Due Date: } \\
9 / 30 / 95\end{array}$ \\
\hline \multicolumn{3}{|c|}{ Control Number: HEP-95-019 } & Revision: 0 \\
\hline $\begin{array}{l}\text { Milestone Type: } \\
\square \text { DOE-HQ } \\
\square \text { DOE-RL } \\
X \text { CNTR }\end{array}$ & $\begin{array}{ll} & \text { Division: } \\
\square & \text { State } \\
\square & \text { Federat } \\
\square & \text { DOE } \\
\square & \text { RCRA } \\
\square & \text { TPA\# } \\
\end{array}$ & $\begin{array}{ll} & \text { DELIVERABLE: } \\
\square & \text { Report } \\
\square & \text { Letter } \\
\square & \text { Drawings } \\
\mathrm{X} & \text { Other } \\
& \text { (specify) } \\
\text { See description }\end{array}$ & $\begin{array}{ll} & \text { ADDRESS TO: } \\
\square & \text { DOE-HQ } \\
X & \text { DOE-RL } \\
\square & \text { Other } \\
& \text { (specify) }\end{array}$ \\
\hline
\end{tabular}

Complete the Engineering for remaining river siren systems, 200 area, 300 area and 400 area.

Description of what constitutes completion of this milestone:

Engineering drawings completed and design documents completed so that sirens can be purchased and installed in 1996.

\begin{tabular}{|ll|lc|}
\hline Cost Account Manager & Date & $\begin{array}{l}\text { Program/Project Manager } \\
\text { JW Tritz }\end{array}$ & Date \\
\hline Program Element Manager & Date & $\begin{array}{l}\text { DOE Monitor } \\
\text { JB Hall }\end{array}$ & Date \\
\hline
\end{tabular}




\section{Tit7e:}

Develop Procedure for combined Supply System/Hanford Emergency

\begin{tabular}{|l|}
\hline Assigned To: JW Tritz \\
\hline WBS Designator: $\quad 6.7 .2 .3$
\end{tabular}

Date Prepared:

$8 / 31 / 94$

CIN:

Due Date:

$9 / 30 / 95$

Control Number: HEP-95-020

Milestone Type:

$\square$ DOE-HQ

$\square$ DOE-RL

$X$ CNTR

\begin{tabular}{|ll} 
& Division: \\
$\square$ & State \\
$\square$ & Federal \\
$\square$ & DOE \\
$\square$ & RCRA \\
$\square$ & TPA\# \\
&
\end{tabular}

Revision: 0

DELIVERABLE:

Report

Letter

Drawings

$X$ other

(specify)

Procedure revised

ADDRESS TO:

$\square$ DOE-HQ

$X$ DOE-RL

$\square$ Other

(specify)

Milestone Description

Revise $\mathrm{plans}$ and procedures to be able to respond to a combined Supply System/Hanford emergency.

\section{Description of what constitutes completion of this milestone:}

RLEP 2.1 revised and issued.

\begin{tabular}{|ll|lr|}
\hline Cost Account Manager & Date & $\begin{array}{l}\text { Program/Project Manager } \\
\text { JW Tritz }\end{array}$ & Date \\
\hline Program Element Manager & Date & $\begin{array}{l}\text { DOE Monitor } \\
\text { JB Hall }\end{array}$ & Date \\
\hline
\end{tabular}


TitTe:

Enhance Site Hazards Assessment and Consequence Assessment Capabilities

\begin{tabular}{|c|c|c|c|}
\hline Assigned To: J & ritz & & CIN: \\
\hline WBS Designator: & 6.7 .2 .3 & & $\begin{array}{l}\text { Due Date: } \\
9 / 30 / 95\end{array}$ \\
\hline Control Number: & EP-95-021 & & Revision: 0 \\
\hline $\begin{array}{l}\text { Milestone Type: } \\
\square \text { DOE-HQ } \\
X \text { DOE-RL } \\
\square \text { CNTR }\end{array}$ & $\begin{array}{ll} & \text { Division: } \\
\square & \text { State } \\
\square & \text { Federat } \\
\square & \text { DOE } \\
\square & \text { RCRA } \\
\square & \text { TPA\# } \\
\end{array}$ & $\begin{array}{ll} & \text { DELIVERABLE: } \\
\square & \text { Report } \\
\square & \text { Letter } \\
\square & \text { Drawings } \\
X & \text { Other } \\
\text { (specify) } \\
\text { See Description }\end{array}$ & $\begin{array}{ll} & \text { ADDRESS T0: } \\
\square & \text { DOE-HQ } \\
X & \text { DOE-RL } \\
\square & \text { Other } \\
\text { (specify) }\end{array}$ \\
\hline
\end{tabular}

Milestone Description

Enhance the Site hazards assessment and consequence assessment capabilities.

Date Prepared:

$8 / 31 / 94$

CIN:

Due Date:

Revision: 0

$\square$ DOE-HQ

DOE-RL

Other

specify)

Description of what constitutes completion of this milestone:

Completion of WHC Milestones HEP-95-022

HEP-95-023

HEP-95-024

\begin{tabular}{|ll|ll|}
\hline Cost Account Manager & Date & $\begin{array}{l}\text { Program/Project Manager } \\
\text { JW Tritz }\end{array}$ & Date \\
\hline Program Element Manager & Date & $\begin{array}{l}\text { DOE Monitor } \\
\text { JB Hall }\end{array}$ & Date \\
\hline
\end{tabular}




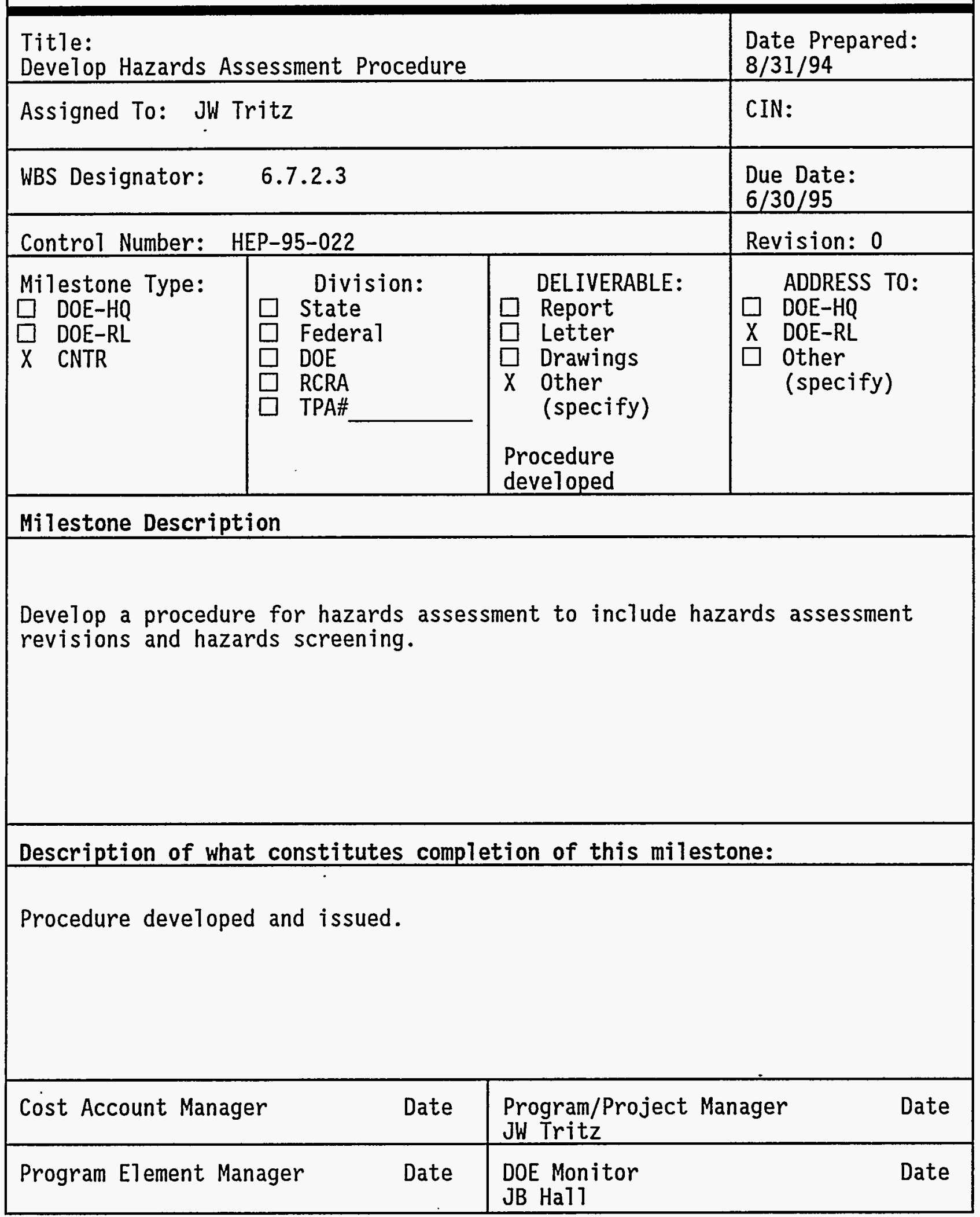




\begin{tabular}{|c|c|c|c|}
\hline \multicolumn{3}{|l|}{$\begin{array}{l}\text { Title: } \\
\text { MESOI Evaluation }\end{array}$} & $\begin{array}{l}\text { Date Prepared: } \\
8 / 31 / 94\end{array}$ \\
\hline \multicolumn{3}{|c|}{ Assigned To: JW Tritz } & CIN: \\
\hline WBS Designator: & \multicolumn{2}{|l|}{6.7 .2 .3} & $\begin{array}{l}\text { Due Date: } \\
9 / 30 / 95\end{array}$ \\
\hline \multicolumn{3}{|c|}{ Control Number: HEP-95-023 } & Revision: 0 \\
\hline $\begin{array}{l}\text { Milestone Type: } \\
\square \text { DOE-HQ } \\
\square \text { DOE-RL } \\
\text { X CNTR }\end{array}$ & $\begin{array}{ll} & \text { Division: } \\
\square & \text { State } \\
\square & \text { Federat } \\
\square & \text { DOE } \\
\square & \text { RCRA } \\
\square & \text { TPA\# }\end{array}$ & $\begin{array}{ll} & \text { DELIVERABLE: } \\
\square & \text { Report } \\
\square & \text { Letter } \\
\square & \text { Drawings } \\
\mathrm{X} & \text { Other } \\
\text { (specify) } & \\
\text { See description }\end{array}$ & $\begin{array}{ll} & \text { ADDRESS TO: } \\
\square & \text { DOE-HQ } \\
X & \text { DOE-RL } \\
\square & \text { Other } \\
\text { (specify) }\end{array}$ \\
\hline
\end{tabular}

Conduct the necessary evaluation to identify required modifications to the MESOI meteorological evaluation computer model to correct for decoupled flow analysis.

Description of what constitutes completion of this milestone:

Evaluation document completed.

\begin{tabular}{|ll|ll|}
\hline Cost Account Manager & Date & $\begin{array}{l}\text { Program/Project Manager } \\
\text { JW Tritz }\end{array}$ & Date \\
\hline Program Element Manager & Date & $\begin{array}{l}\text { DOE Monitor } \\
\text { JB Hall }\end{array}$ & Date \\
\hline
\end{tabular}




\begin{tabular}{|c|c|c|c|}
\hline \multicolumn{3}{|c|}{$\begin{array}{l}\text { Title: } \\
\text { Procure EMS Phase II Equipment }\end{array}$} & $\begin{array}{l}\text { Date Prepared: } \\
8 / 31 / 94\end{array}$ \\
\hline \multicolumn{3}{|c|}{ Assigned To: JW Tritz } & CIN: \\
\hline WBS Designator: & 6.7 .2 .3 & & $\begin{array}{l}\text { Due Date: } \\
9 / 30 / 954\end{array}$ \\
\hline \multicolumn{3}{|c|}{ Control Number: HEP-95-024 } & Revision: 0 \\
\hline $\begin{array}{l}\text { Milestone Type: } \\
\square \text { DOE-HQ } \\
\square \text { DOE-RL } \\
\text { X CNTR }\end{array}$ & $\begin{array}{ll} & \text { Division: } \\
\square & \text { State } \\
\square & \text { Federal } \\
\square & \text { DOE } \\
\square & \text { RCRA } \\
\square & \text { TPA\# }\end{array}$ & $\begin{array}{ll} & \text { DELIVERABLE: } \\
\square & \text { Report } \\
\square & \text { Letter } \\
\square & \text { Drawings } \\
\mathrm{X} & \text { Other } \\
& \text { (specify) } \\
& \\
\text { Equipment } \\
\text { procured }\end{array}$ & $\begin{array}{ll} & \text { ADDRESS TO: } \\
\square & \text { DOE-HQ } \\
X & \text { DOE-RL } \\
\square & \text { Other } \\
& \text { (specify) }\end{array}$ \\
\hline
\end{tabular}

Identify the necessary EMS Phase II equipment and software and procure.

Description of what constitutes completion of this milestone:

Phase II equipment identified in plan and procured to install in Site emergency centers.

\begin{tabular}{|ll|lc|}
\hline Cost Account Manager & Date & $\begin{array}{l}\text { Program/Project Manager } \\
\text { JW Tritz }\end{array}$ & Date \\
\hline Program Element Manager & Date & $\begin{array}{l}\text { DOE Monitor } \\
\text { JB Ha } 7\end{array}$ & Date \\
\hline
\end{tabular}


Westinghouse Hanford Company MILESTONE DESCRIPTION SHEET

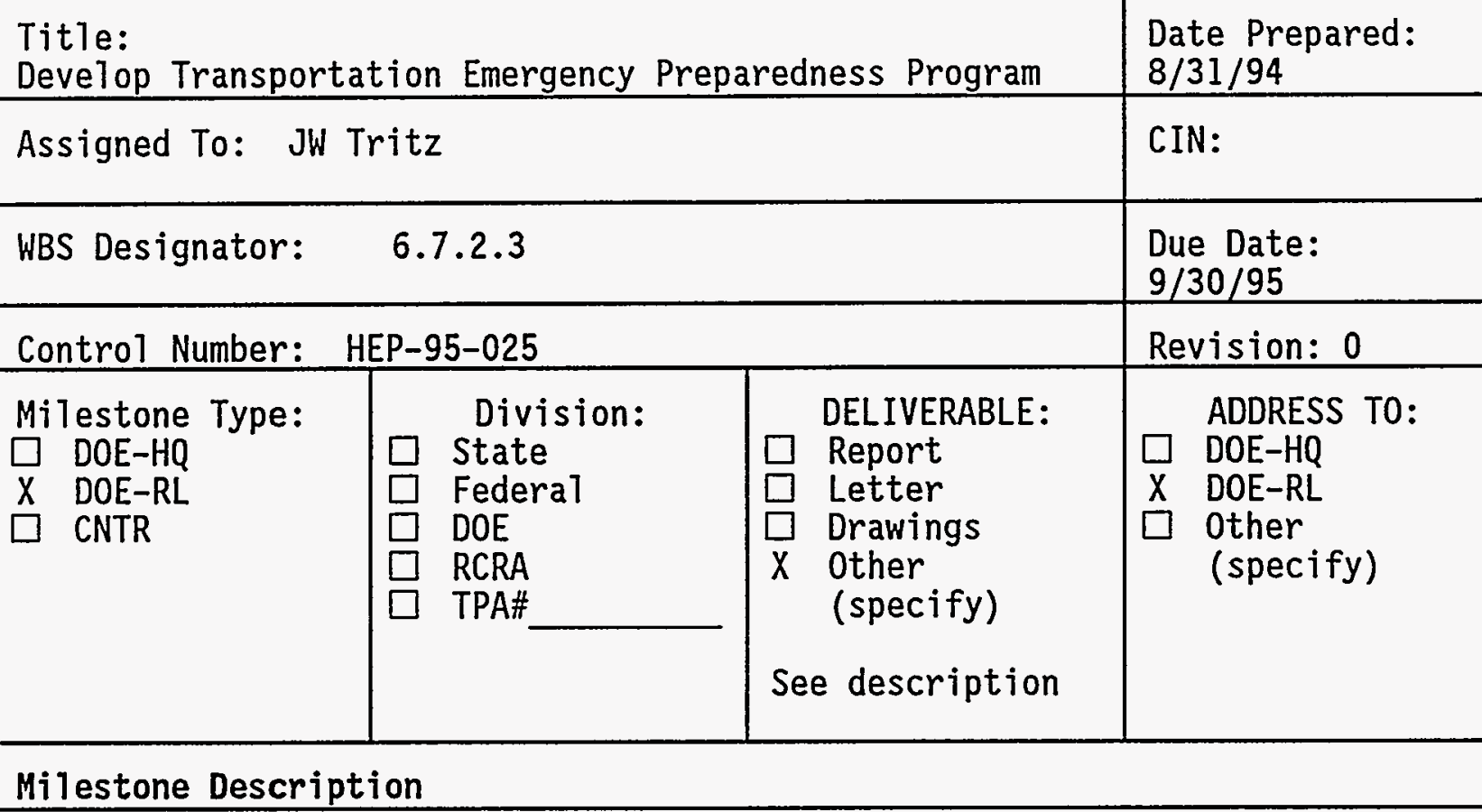

Develop a Transportation Emergency Preparedness Program.

Description of what constitutes completion of this milestone:

Completion of WHC Milestones HEP-95-026

HEP-95-027

\begin{tabular}{|ll|lc|}
\hline Cost Account Manager & Date & $\begin{array}{l}\text { Program/Project Manager } \\
\text { JW Tritz }\end{array}$ & Date \\
\hline Program Element Manager & Date & $\begin{array}{l}\text { DOE Monitor } \\
\text { JB Hall }\end{array}$ & Date \\
\hline
\end{tabular}




\begin{tabular}{|c|c|c|c|}
\hline \multicolumn{3}{|c|}{$\begin{array}{l}\text { Title: } \\
\text { Develop a Transportation Emergency Preparedness Plan }\end{array}$} & $\begin{array}{l}\text { Date Prepared: } \\
8 / 31 / 94\end{array}$ \\
\hline \multicolumn{3}{|c|}{ Assigned To: JW Tritz } & CIN: \\
\hline WBS Designator: & \multicolumn{2}{|l|}{0.7 .2 .3} & $\begin{array}{l}\text { Due Date: } \\
6 / 30 / 95\end{array}$ \\
\hline \multicolumn{3}{|c|}{ Control Number: HEP-95-026 } & Revision: 0 \\
\hline $\begin{array}{l}\text { Milestone Type: } \\
\square \text { DOE-HQ } \\
\square \text { DOE-RL } \\
\times \text { CNTR }\end{array}$ & $\begin{array}{ll} & \text { Division: } \\
\square & \text { State } \\
\square & \text { Federa } \\
\square & \text { DOE } \\
\square & \text { RCRA } \\
\square & \text { TPA\# }\end{array}$ & $\begin{array}{ll} & \text { DELIVERABLE: } \\
\square & \text { Report } \\
\square & \text { Letter } \\
\square & \text { Drawings } \\
X & \text { Other } \\
& \text { (specify) } \\
& \\
\text { Plan issued }\end{array}$ & $\begin{array}{ll} & \text { ADDRESS TO: } \\
\square & \text { DOE-HQ } \\
X & \text { DOE-RL } \\
\square & \text { Other } \\
& \text { (specify) }\end{array}$ \\
\hline
\end{tabular}

Develop a Transportation Emergency Preparedness Plan.

Description of what constitutes completion of this milestone:

Transportation Emergency Preparedness Plan issued.

\begin{tabular}{|ll|lc|}
\hline Cost Account Manager & Date & $\begin{array}{l}\text { Program/Project Manager } \\
\text { JW Tritz }\end{array}$ & Date \\
\hline Program El ement Manager & Date & $\begin{array}{l}\text { DOE Monitor } \\
\text { JB Hall }\end{array}$ & Date \\
\hline
\end{tabular}


Title:

Develop and Conduct a Transportation Exercise

Date Prepared:

$8 / 31 / 94$

\begin{tabular}{|c|c|c|c|}
\hline Assigned To: J & ritz & & CIN: \\
\hline WBS Designator: & 6.7 .2 .3 & & $\begin{array}{l}\text { Due Date: } \\
9 / 30 / 95\end{array}$ \\
\hline Control Number: & EP-95-027 & & Revision: 0 \\
\hline $\begin{array}{l}\text { Milestone Type: } \\
\square \text { DOE-HQ } \\
\square \text { DOE-RL } \\
\text { X CNTR }\end{array}$ & \begin{tabular}{ll}
\multicolumn{1}{c}{ Division: } \\
$\square$ State \\
$\square$ Federal \\
$\square$ DOE \\
$\square$ RCRA \\
$\square$ TPA\# \\
\end{tabular} & $\begin{array}{ll} & \text { DELIVERABLE: } \\
\square & \text { Report } \\
\square & \text { Letter } \\
\square & \text { Drawings } \\
\mathrm{X} & \text { Other } \\
& \text { (specify) } \\
& \\
\text { Exercise } \\
\text { completed }\end{array}$ & $\begin{array}{ll} & \text { ADDRESS TO: } \\
\square & \text { DOE-HQ } \\
X & \text { DOE-RL } \\
\square & \text { Other } \\
& \text { (specify) }\end{array}$ \\
\hline
\end{tabular}

Milestone Description

Develop and conduct a transportation exercise

Description of what constitutes completion of this milestone:

Exercise completed.

\begin{tabular}{|ll|lc|}
\hline Cost Account Manager & Date & $\begin{array}{l}\text { Program/Project Manager } \\
\text { JW Tritz }\end{array}$ & Date \\
\hline Program Element Manager & Date & $\begin{array}{l}\text { DOE Monitor } \\
\text { JB Hall }\end{array}$ & Date \\
\hline
\end{tabular}

